\title{
Evolution of resistance to COVID-19 vaccination with dynamic lockdown
}

\author{
Martin Nowak ( $\square$ martin_nowak@harvard.edu ) \\ Harvard University https://orcid.org/0000-0001-5489-0908

\section{Gabriela Lobinska} \\ Weizmann Institute of Science

\section{Ady Pauzner} \\ Tel Aviv University

\section{Arne Traulsen} \\ Max Planck Institute for Evolutionary Biology https://orcid.org/0000-0002-0669-5267

\section{Yitzhak Pilpel} \\ Weizmann Institute of Science
}

\section{Article}

Keywords: COVID-19, pandemic, vaccinations, immunology

Posted Date: July 13th, 2021

DOI: https://doi.org/10.21203/rs.3.rs-646895/v1

License: (c) (i) This work is licensed under a Creative Commons Attribution 4.0 International License. Read Full License

Version of Record: A version of this preprint was published at Nature Human Behaviour on February 24th, 2022. See the published version at https://doi.org/10.1038/s41562-021-01281-8. 
1 Evolution of resistance to COVID-19 vaccination with dynamic lockdown

3 Gabriela Lobinska*1, Ady Pauzner ${ }^{2}$, Arne Traulsen ${ }^{3}$, Yitzhak Pilpel*1, Martin A Nowak*4

41 Department of Molecular Genetics, Weizmann Institute of Science, Rehovot 76100,

5 Israel

62 Berglas School of Economics, Tel Aviv University, Tel Aviv 69978, Israel

73 Department of Evolutionary Theory, Max-Planck-Institute for Evolutionary

8 Biology, August-Thienemann-Str. 2, 24306 Ploen, Germany

94 Department of Mathematics, Department of Organismic and Evolutionary Biology,

10 Harvard University, Cambridge MA 02138, USA

The COVID-19 pandemic has led to an unprecedented global response in terms of social

13 lockdown in order to slow the spread of the virus ${ }^{1,2}$. Currently the greatest hope is

14 based on world-wide vaccination ${ }^{3,4}$. The expectation is that social and

15 economic activities can gradually resume as more and more people become vaccinated.

16 Yet, a relaxation of social distancing that allows increased transmissibility, coupled with

17 selection pressure due to vaccination, will likely lead to the emergence of vaccine

18 resistance ${ }^{5}$. Here we analyze the evolutionary dynamics of COVID-19 in the presence of

19 dynamic lockdown and in response to vaccination. We use infection and vaccination

20 data of 6 different countries (Israel, US, UK, Brazil, France and Germany) to assess the

21 probability and timing for the wave of vaccine resistant mutant ${ }^{2}$. For slow vaccination

22 rates, resistant mutants will appear inevitably even if strict lockdown is maintained. For

23 fast vaccination rates (such as those used in Israel) the emergence of the mutant can be

24 prevented if strict lockdown is maintained during vaccination. Our mathematical results

25 provide quantitative guidelines for a combined vaccination and lockdown policy that

26 minimizes the probability of emergence of vaccine resistance variants for current and

27 future vaccination programs. 
29 The COVID-19 pandemic has had a devastating effect on global health and economy. Since

30 the identification of the first SARS-COV-2 case in December 2019, 178.71 million

31 infections have been recorded and at least 3.86 million people have died as a result of the

32 infection (as of June 2021) 2 $^{2}$ The increased mortality and complication rates of SARS-COV-

$332^{1}$ compared to the mild diseases caused by seasonal coronaviruses, such as HCoV-229E 6 ,

34 have led to unparalleled governmental and individual-level responses in order to reduce

35 the number of SARS-COV-2 infections.

36 Since the beginning of the pandemic, it has become clear that non-pharmaceutical

37 interventions (NPI), such as lockdowns, are economically and socially unsustainable in the

38 long run. Periodical loosening and tightening of social distancing measures, which present

39 an attempt at balancing economical and sanitary considerations, have led to waves of

40 increase and decrease in the number of SARS-COV-2 infections per day ${ }^{2}$ (see Figure 1A).

41 Therefore, much hope has been placed on vaccine development, which would allow the

42 immunization of a large fraction of the population, thereby substantially reducing

43 mortality and potentially achieving herd immunity, which could in principle eradicate

44 SARS-COV-2 altogether.

45 Mass vaccination campaigns have been launched in many countries (see Figure 1B), most

46 notably Israel and the UK (both more than $60 \%$ of vaccinated population) and the US and

47 Germany (both more than $50 \%$ of vaccinated population). Currently, four companies are

48 producing vaccines that have been approved for emergency use either by the Food and

49 Drug Administration $(F D A)^{3}$ or by the European Medicines Agency (EMA) ${ }^{4}$ : Pfizer-

50 Biontech, Moderna, AstraZeneca and Johnson \& Johnson/Janssen Pharmaceuticals.

51 Several other vaccines are also used outside of the European Union and the USA:

52 Gamaleya (Sputnik V), Sinopharm Beijing, Sinovac, Sinopharm-Wuhan and Bharat-Biotech

53 (Covaxin $)^{7}$.

54

55 

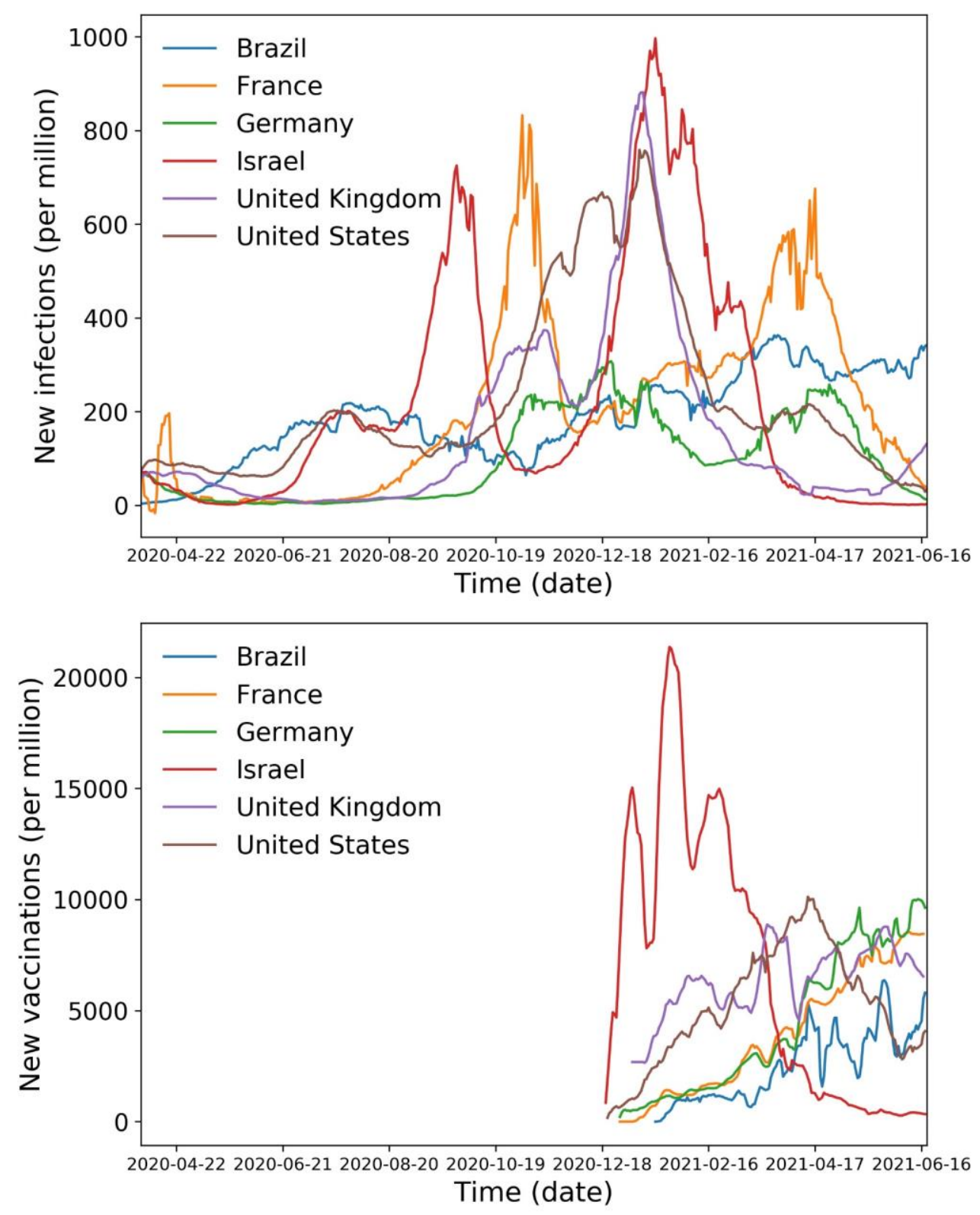

58 Figure 1: New SARS-CoV2 cases per day per million and number of vaccinations against SARS-CoV2 per 59 day per million in France, Germany, USA, Israel, Brazil and UK. (A) In an attempt to balance economic and 60 sanitary considerations, these six countries have gone through several cycles of loosening and tightening 61 government-imposed restrictions, resulting in periodical increases and decreases in the number of SARS-

62 CoV2 infections per day. The so-called "British variant", identified in November 2020, is most probably 63 responsible for the increase in the number of infections in the UK and Israel at that time. (B) Large scale 64 vaccination programs commenced in December 2020. At the peak, Israel vaccinated more than 20,000 65 people per million (2\%) per day. The vaccination rate then decreased in April 2021 as most eligible 66 individuals had been vaccinated. 
67 However, the identification of new SARS-COV-2 variants has cast a shadow over the expectation of a swift end of the pandemic ${ }^{8,9}$. The so-called "British" variant (B.1.1.7), now termed $\alpha$, and "South African" variant (501.V2), now termed $\beta$, have been shown to be neutralized to a lesser extent by convalescent and vaccinee sera ${ }^{10}$, although experiments on non-human primates have shown that this decrease might not necessarily cause a decrease in immunity ${ }^{11}$. Structural studies have mapped and

73 predicted mutations that lead to antibody escape ${ }^{12-14}$. As vaccination around the world progresses, the continued evolution of SARS-COV-2 could eventually give rise to a fully vaccine resistant variant. Such a variant could quickly spread due to its ability to infect vaccinated and recovered in addition to fully susceptible individuals. The question of emergence of vaccine resistance has already been the subject of many research papers ${ }^{15-}$ 18 .

What policy could be exercised that would minimize the chance of emergence of vaccine resistant strains? Policymakers can vary the extent of social distancing imposed and regimes of vaccine administration. The critical biological parameters on the other hand include the infectivity of the various strains and the rate of mutation of the virus that may ultimately lead to emergence of a resistant strain. Here we introduce a mathematical model that examines various combinations of these parameters. Our model helps to design optimal policies that would minimize the chance of emergence of resistant strains or maximize the time until their occurrence.

87 Our paper is an addition to the extensive body of work that has been performed in the past year in order to understand the spread and evolution of SARS-COV-2 ${ }^{19-25}$. SARS-COV2 research has drawn on a very long history of epidemiological research $26,27,36-38,28-35$. Due to the global and urgent nature of the pandemic, many studies that could inform policy91 making have been conducted ${ }^{5,39,48,40-47}$.

92 In order to understand the evolutionary potential of the virus in response to a vaccination 93 program we study a stochastic model for infection dynamics and virus evolution in the 94 presence of varying degrees of social lockdown and different vaccination rates. We 
95 distinguish between a wild-type virus (WT) and a vaccine resistant mutant virus (MT). The 96 vaccine is effective against the WT strain, while the MT strain evades immunity induced 97 by the vaccine either partially or completely. We build upon the mathematical framework of the Susceptible-Infected-Removed (SIR) model from epidemiology ${ }^{32,49}$, albeit with 99 considerable adjustments necessitated by the specific problem at hand. Our model keeps track of people who are susceptible, infected by WT or MT, recovered from WT or MT, vaccinated or unvaccinated (Figure 2).

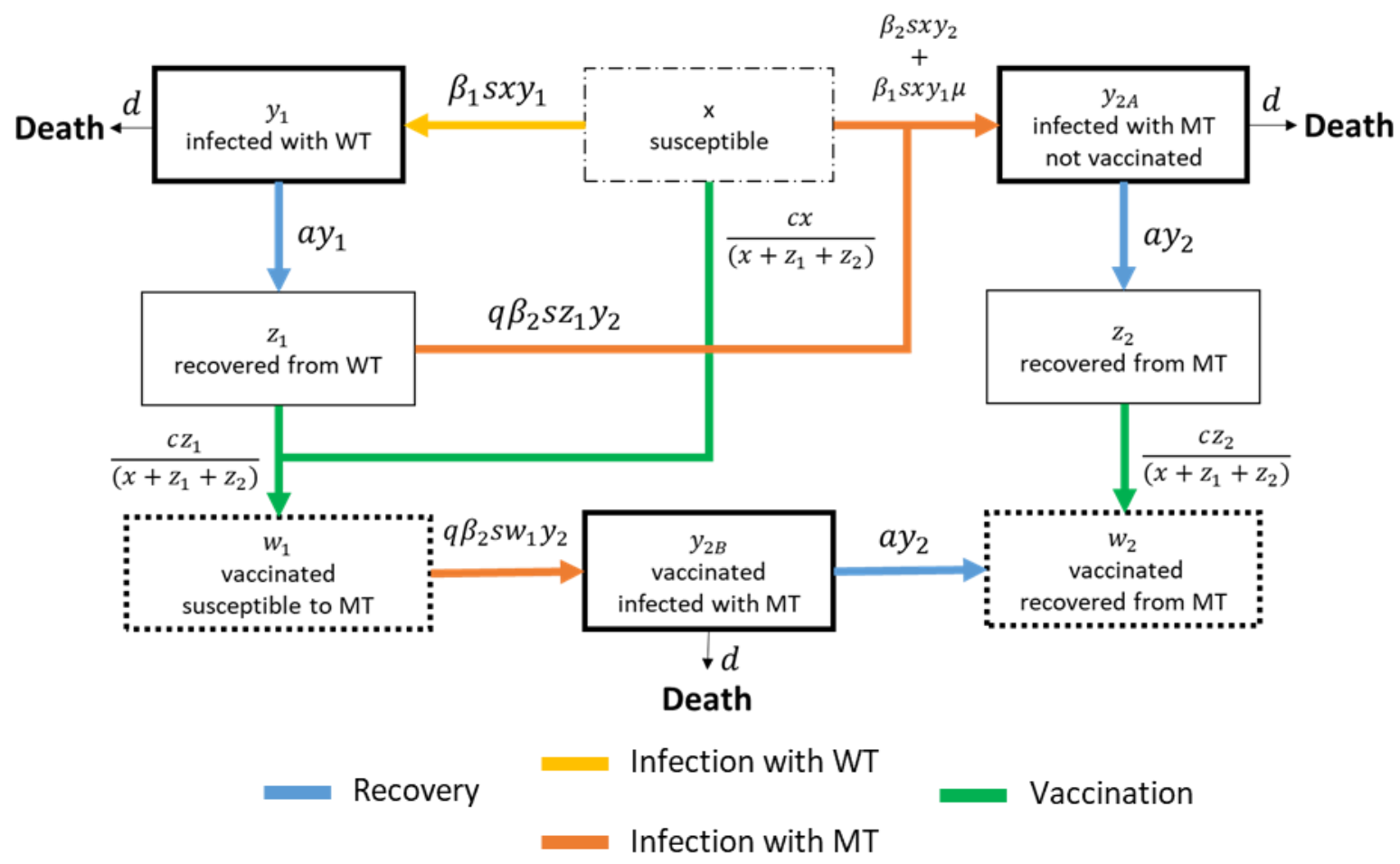

Figure 2: Infection dynamics, vaccination and resistance. Susceptible individuals $(x)$ can be infected by wildtype (WT) or mutant (MT) virus. Infected people $\left(y_{1}, y_{2 A}, y_{2 B}\right)$ can die (with rate $d$ ) or recover (with rate a). People recovered from WT or vaccinated against WT can be infected by MT. People recovered from MT cannot be infected by WT. We assume equal infectivity, recovery and death rates for both WT and MT. Vaccination occurs at rate $c$ per day for all unvaccinated individuals (excluding those that are currently in active infection). Mutation happens (at rate $\mu$ ) when exposure to a WT infected individual $\left(y_{1}\right)$ results in the generation of a MT infected individual. Since $\mu$ is small, we neglect the term $1-\mu$. The rates of these events are indicated on the arrows and are used in the Gillespie algorithm implementing the stochastic dynamics. 
112 Crucially, we assume there is a dynamic lockdown guided by the number of new infections

113 per day. As that number exceeds a threshold, governmental rules and individual

114 responses reduce social activity. If the number of new infections falls below this

115 threshold, the lockdown is somewhat relaxed and some people stop following the rules,

116 thereby allowing higher transmission of the virus. We simulate these dynamics as a

117 stochastic process. In consequence, we obtain fluctuating numbers of new infections per

118 day. We introduce mass vaccination at alternative fixed rates. Then we compute the

119 probability and timing of the wave of infection caused by the spontaneous emergence of

120 a vaccine resistant virus.

121 In our approach, the mutation rate $\mu$ denotes the probability that a WT-infected individual

122 will infect a susceptible individual with the MT strain. The exact value of this probability

123 is currently unknown and complex to obtain empirically. For the simulations and

124 calculations reported in this paper we therefore consider a wide range of mutation rates.

125 From our model, we also derive an upper bound for the mutation rate using the fact that

126 no wave of a vaccine-resistant variant has occurred up until now. Note that this rate can

127 be very different from the per-base mutation rate of SARS-COV-2, which is about $10^{-5}$.

129 DYNAMICS OF VIRAL INFECTION AND EVOLUTION

130 Our model keeps track of eight different variable states: individuals who are susceptible

$131(x)$, infected with WT $\left(y_{1}\right)$, non-vaccinated and infected with MT $\left(y_{2 A}\right)$, vaccinated and

132 infected with $\mathrm{MT}\left(y_{2 B}\right)$, recovered from WT $\left(z_{1}\right)$, recovered from $\mathrm{MT}\left(z_{2}\right)$, vaccinated but

133 susceptible to $\mathrm{MT}\left(w_{1}\right)$, vaccinated and recovered from $\mathrm{MT}\left(w_{2}\right)$; see Figure 2.

134 The WT strain can infect susceptible individuals $(x)$, converting them to individuals 135 infected with WT $\left(y_{1}\right)$ at rate $\beta_{1}$. A mutation can occur with probability $\mu$. In this case, a 136 WT infected individual infects a susceptible individual $(x)$ with a mutated version of the 137 virus, in a mutation that have taken place in the infecting individual, thus converting the 138 susceptible to a MT infected individual $\left(y_{2 A}\right)$. WT infected individuals either recover with 
rate $a$ and become immune to future WT infection $\left(z_{1}\right)$ or die at rate $d$. Susceptible individuals $(x)$ and individuals recovered from WT $\left(z_{1}\right)$ can become vaccinated individuals

$141\left(w_{1}\right)$. The parameter $c$ denotes the number of individuals vaccinated per day. Hence, the rates of vaccination of $x, z_{1}$ and $z_{2}$ are respectively $c x /\left(x+z_{1}+z_{2}\right), c z_{1} /\left(x+z_{1}+z_{2}\right)$

143 and $c z_{2} /\left(x+z_{1}+z_{2}\right)$. For simplicity we assume single-dose vaccination; for a double 144 dose vaccine our model would describe the application of the second dose ignoring partial 145 immunity caused by the first dose; extension of our model to a full two dose vaccination 146 protocol is straightforward.

147 At rate $\beta_{2}$, the MT strain infects susceptible individuals $(x)$, WT recovered individuals $\left(z_{1}\right)$ 148 and vaccinated individuals who are not immune to MT $\left(w_{1}\right)$. MT infected individuals either 149 recover with rate $a$ and become immune to future MT and WT infection $\left(z_{2}\right)$ or die at 150 same death rate $d$ as with the WT strain, i.e. assuming no difference in lethality between 151 the two strains. We assume one-way cross-immunity induced by the viral strains: the MT 152 strain can infect individuals that have recovered from WT or that have been vaccinated 153 against WT, but the WT strain cannot infect individuals that have recovered from the MT. 154 In practice, the WT strain becomes extinct soon after the appearance of the MT strain, 155 meaning that the number of individuals recovered from MT that could become infected 156 with WT is negligible. We note that our MT strain escapes both from the immunity that is 157 induced by natural infection with WT and the immunity induced by vaccination against 158 WT.

159 We need to distinguish between MT infected individuals that are or are not vaccinated: $160 y_{2 B}$ and $y_{2 A}$, respectively. Upon recovery the former will not be vaccinated (again), while 161 the latter will be vaccinated.

162 We also study partial immunity to the MT strain which can be acquired by recovery from 163 WT infection or by vaccination. For partial immunity, the corresponding infection rates 164 are multiplied by a parameter $q$, which is between 0 and 1 . If $q=1$ then WT infection or 165 vaccination confers no immunity to MT at all; the mutant escapes completely. For $0<$ $166 q<1$, the MT is a partial escape mutant. For $q=0$, the MT does not escape at all. 
167 Lockdown measures are implemented by multiplying the infectivity coefficients of each

168 strain by a social activity parameter $s$ which ranges in $[0,1]$. Unconstrained social 169 interaction means $s=1$, while $s=0$ would be complete lockdown. The population 170 tolerates a certain number of new infections, $L$, per day. Each day, if the number of new 171 infections exceeds $L$, then $s$ is decreased by a random, uniformly distributed number 172 between 0 and 0.1 . If the number of new infections is less than $L$, then $s$ is increased by 173 a random, uniformly distributed number between 0 and 0.1 . In any case, $s$ cannot 174 decrease below 0.05 or increase above 1.

175 As an example, the rate of infection of the recovered from WT $z_{1}$ by the MT strain infected 176 individuals $y_{2}$ is multiplied both by the lockdown coefficient $s$ and the partial immunity 177 coefficient $q$ - hence this rate is given by $q \beta_{2} s w_{1} y_{2}$.

\section{COMPUTATIONAL IMPLEMENTATION AND DATA}

179 A Gillespie algorithm is commonly used to simulate stochastic systems with high variation 180 in waiting times between consecutive events ${ }^{50-53}$. In our model the population is represented as a vector of length eight, corresponding to the eight categories. The rates 182 of all possible events (infection, recovery, death, mutation and vaccination) are 183 calculated. The time of the next event in the model is drawn from an exponential 184 distribution, with parameter dependent on the sum of all event rates and an event is 185 chosen, with probability proportional to its rate. The population is updated according to 186 the event that occurred. The simulation is stopped when there are no more infected 187 individuals in the population. The algorithm is presented in pseudocode in the Appendix, 188 along with a table of the possible events of the model and their default rates.

189 In order to achieve feasible computation time and resources, we simulated populations 190 of size up to $N=10^{6}$. The results of those simulations can be scaled to larger population 191 sizes by considering a population of for example $N=10^{7}$ as $m=10$ "batches" of $10^{6}$ 192 individuals, and computing the results for $N=10^{7}$ as $1-(1-p)^{m}$, where $p$ is the 193 proportion of runs where the MT strain took over. Figure S1 shows the strong agreement 
194 between simulated results and the results scaled from simulations with smaller 195 population sizes.

196 For all our simulations, we have endeavored to use real world data for all model 197 parameters. In particular, infection and vaccination data has been obtained from the 198 database Our World in Data ${ }^{2}$ (OWID) and downloaded on June 19 2021.

199 In our simulation, since the number of new infections each day is constant, the number 200 of susceptible individuals decreases linearly with slope $-L / a$. Vaccination of both 201 susceptible and recovered individuals proceeds at rate $c$. The social activity parameter, $s$, 202 increases as more and more individuals become immunized either by infection or by 203 vaccination. The WT reproductive rate, $R_{W T}$, is maintained at 1 as long as the MT has not 204 appeared. The MT reproductive rate, $R_{M T}$, increases with the social activity parameter $S$ 205 until the MT strain takes over. After MT takeover, the MT reproductive rate $R_{M T}$ is 206 buffered at 1 by the dynamic lockdown (see Figure 3 and Methods). 

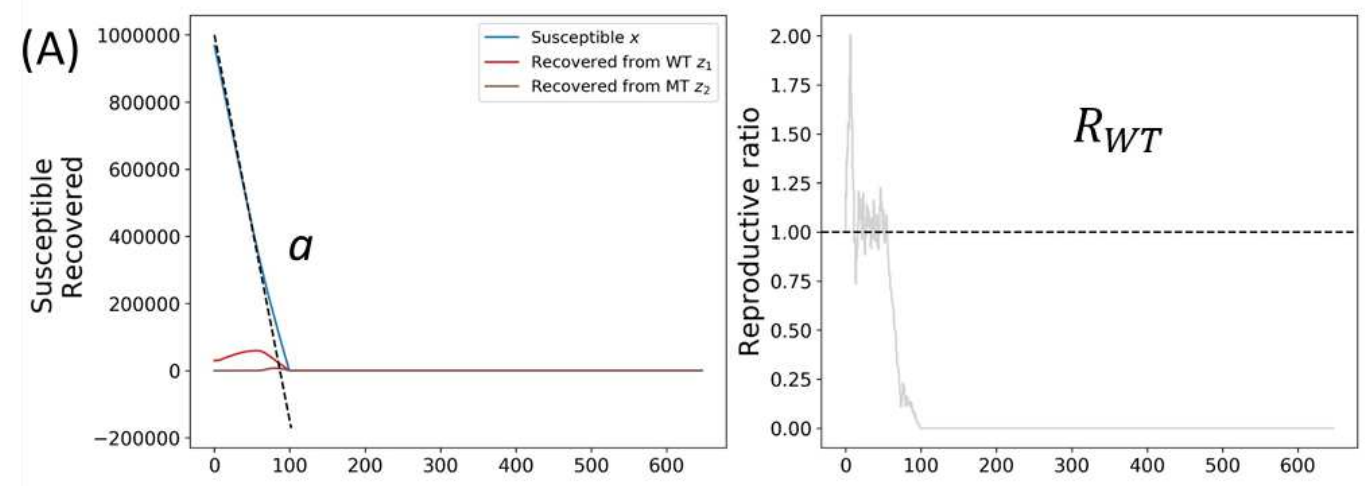

(B)
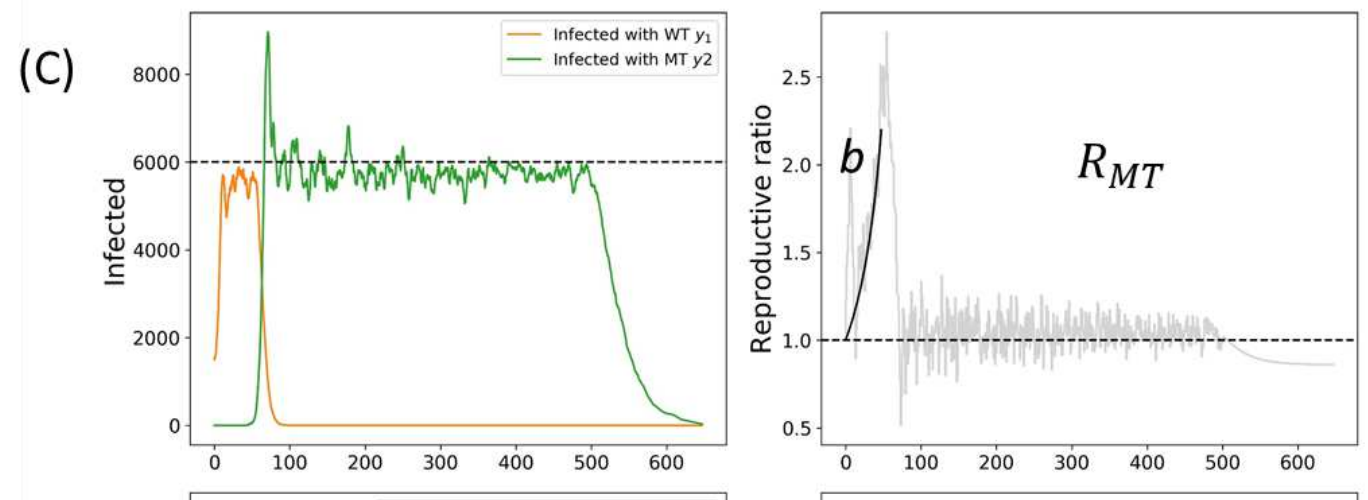

(D)
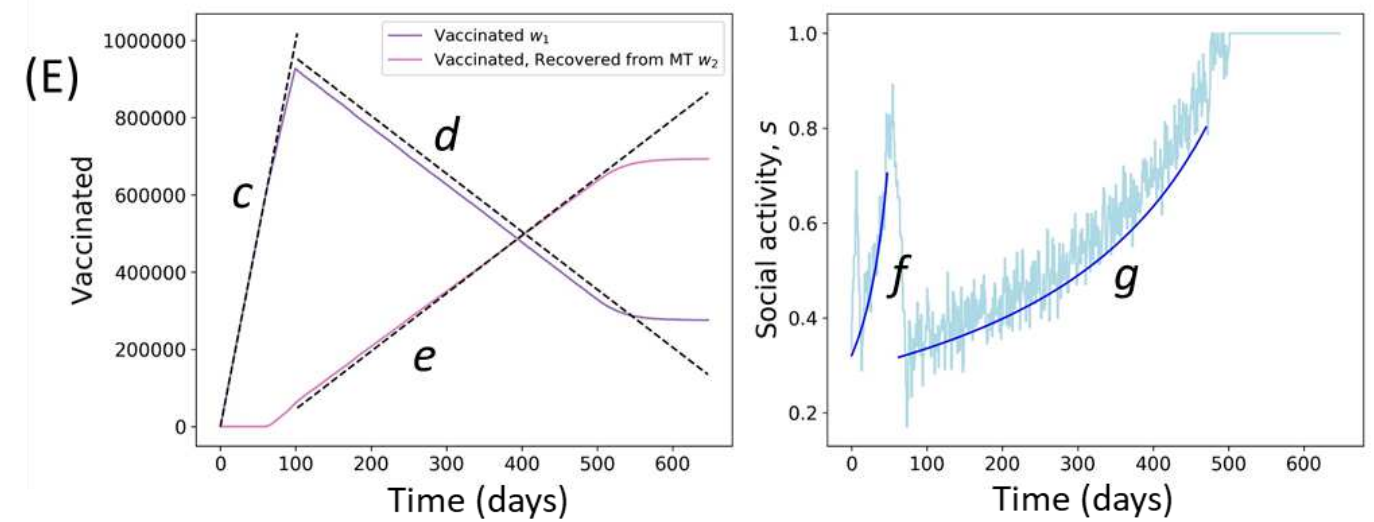

(F)

Figure 3: Evolution of resistance in presence of vaccination. (A) Before MT takeover, the decline in susceptible individuals $(x)$ can be approximated by a linear function with slope equal to the vaccination rate $c$. Since vaccination is fast, individuals recovered from WT and non-vaccinated individuals recovered from MT are few. The equation of line (a) is $x(t)=x(0)-c t$ for $t<t^{*}$ where $t^{*}$ is the time of takeover of the MT. (B) The reproductive rate $R_{W T}$ is maintained at around 1 by dynamic lockdown. After mutant takeover,

$213 R_{W T}$ is less than 1 , since the lockdown is now adjusted to the population susceptible to the MT strain. (C)

214 The number of active WT infections before takeover and of active MT infections after takeover, is 215 fluctuating around $L / a$ until herd immunity to the MT is reached. (D) Before MT takeover, the reproductive 216 rate of the MT grows as (b) $R_{M T}=\beta_{2}\left(x(t)+z_{1}(t)+w_{1}(t)\right) / a$. After takeover, $R_{M T}$ is maintained around 1. (E) The number of vaccinated individuals $\left(w_{1}\right)$ first increases linearly with slope equal to the vaccination rate. After MT takeover, the number of individuals vaccinated to the WT and recovered from MT $\left(w_{2}\right)$ 
219 increased linearly with slope $L$. The equations of the lines are given by (c) $w_{1}(t)=c t$ for $t<t^{*}(\mathrm{~d}) w_{1}(t)=$ $220 w_{1}\left(t^{*}\right)-L\left(t-t^{*}\right)$ for $t>t^{*} \quad$ (e) $w_{2}(t)=L\left(t-t^{*}\right)$ for $>t^{*}$. (F) Before MT takeover, the dynamic 221 lockdown is adjusted to the WT. As the number of individuals immune to WT grows, social activity increases.

222 When the MT emerges, lockdown measures are reinstated. Subsequently, social activity increases as the 223 population immune to the MT grows. The equations for the lines given by (f) $s(t)=a / \beta_{1} x(t)$ for $t<t^{*}$ $224 ;(\mathrm{g}) s(t)=a / \beta_{2}\left(x(t)+z_{1}(t)+w_{1}(t)\right)$ for $>t^{*}$.

225 We performed 1000 runs of the stochastic simulation for each combination of parameters 226 reflecting realistic values of the two model parameters determined by governmental 227 policy: the tolerated number of infections per day, $L$, and the vaccination rate per day, $c$. 228 Each square of the color map shown in Figure 4 reflects the average value of these 1000 229 runs, which were performed for a population of $N=10^{6}$ and then scaled to $N=10^{7}$ 230 and $N=10^{8}$. At each combination of $L$ and $c$ the color maps denote the predicted 231 probability of a mutant take over. We perform computations using $q=1$ and $q=0.4$ for 232 complete and partial immune evasion by the mutant. 


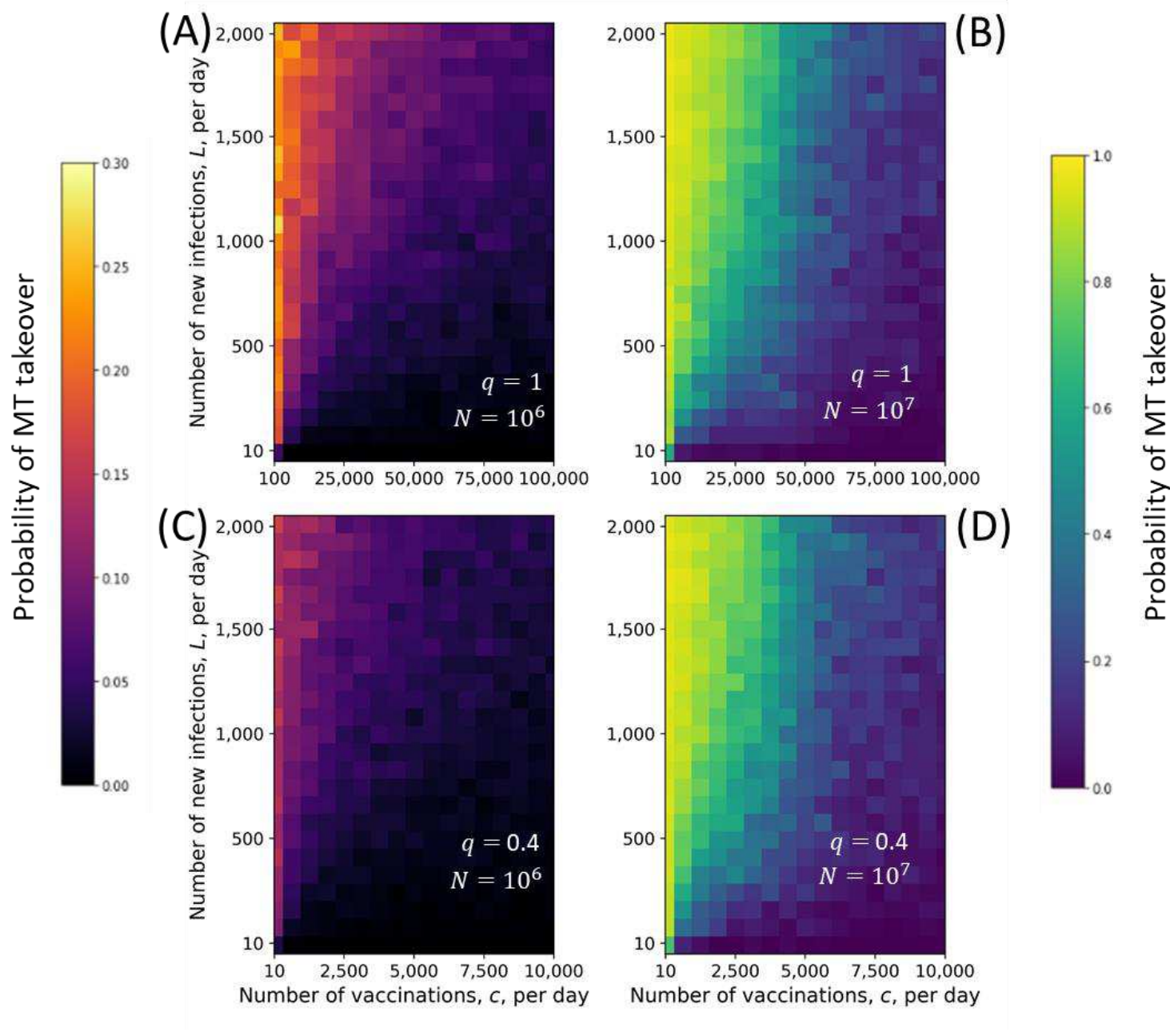

234 Figure 4: Probability of emergence of resistance. For each square of the color maps, the proportion of runs

235 (out of 1000 runs) where the number of individuals infected with the MT strain exceeded the number of

236 individuals infected with the WT strain is recorded. All simulations are run for a population size of $N=10^{6}$,

237 then scaled to obtain the results shown for $N=10^{7}$. Results for color maps (B) and (D) were scaled 238 according to $\left(1-(1-p)^{10}\right)$, where $p$ is the proportion of runs where the MT strain took over. We observe 239 a triangular shape of $(L, c)$ parameter sets for which the MT strain takes over, indicating that high 240 vaccination rates can be safely associated with more lenient social distancing measures. On the other hand, 241 very slow vaccination cannot be compensated by any strength of lockdown. Partial immunity to the WT 242 strain (panels (A) and (C)) does not affect the shape of the parameter space where we observe MT takeover, 243 but reduces its probability. 
245 Allowing a large amount of infection cases and slow vaccination results is almost certain 246 takeover of the MT strain. On the other hand, very fast vaccination coupled with a low 247 number of tolerated new infections per day can prevent emergence of the MT. Partial 248 immune evasion ( $q=0.4$ ) of the mutant slightly reduces the probability of its takeover.

249 Note that the shape of the parameter space where we observe takeover is similar for $q=$ $250 \quad 1$ and $q=0.4$.

REPRODUCTIVE RATIO OF THE MUTANT AND PROBABILITY OF TAKEOVER

252 In Figure 5 we show detailed data from 6 countries together with the estimated 253 reproductive ratio, $R_{M T}$, of a vaccine resistant mutant and the probability of generating a wave of resistant virus. Data for the number of susceptible individuals $x(t)$, vaccinated 255 individuals $w(t)$, recovered individuals $z(t)$, newly infected individuals $L(t)$, and an 256 estimate for the reproductive rate $R_{W T}$ of the WT can be obtained from OWID ${ }^{2}$. The 257 reproductive rate $R_{M T}$ of the escape mutant can be calculated according to:

$$
R_{M T}(t)=R_{W T}(t)[x(t)+q w(t)+q z(t)] / x(t)
$$

258 The probability of not producing an escape mutant in a given day is $(1-\mu)^{L(t)}$. The 259 probability of not producing a surviving escape mutant is $(1-\rho(t) \mu)^{L(t)}$, where $\rho(t)$ is 260 the survival probability of a mutant generated on that day. If $R_{M T}(t)<1$ then $\rho(t)=0$. 261 If $R_{M T}(t)>1$ we assume $\rho(t)=1-1 / R_{M T}(t)$. The probability that no surviving mutant 262 is generated between time 0 and time $t$ is given by

$$
P(t)=\prod_{\tau=0}^{t}[1-\mu \rho(\tau)]^{L(\tau)}
$$

263 In Figure 5 we show the reproductive rate of the mutant $R_{M T}(t)$ and the probability $P(t)$ 264 of generating a surviving escape mutant as a function of time. Prior to vaccination the 265 reproductive rate of a potential escape mutant tracks closely that of the WT. As people 266 become vaccinated in large numbers, $R_{M T}$ starts to increase significantly above $R_{W T}$. 267 Nevertheless, it is possible to keep $R_{M T}$ below one by maintaining some measures of 268 lockdown. (This is the case for Israel and UK). Overall, the probability that Israel generated 269 a vaccine escape mutant (before June 2021) is of order of 1-2 percent (assuming $\mu=$ 
$27010^{-7}$ ). For the same mutation rate the corresponding probability for the United States is

27132 percent; the United States have a much larger total population size but also many more

272 infections per million people. The corresponding probabilities for Brazil, France, Germany,

273 and UK are 17, 8, 4 and 6 percent (see Table 1).

274

275

276

277

278 

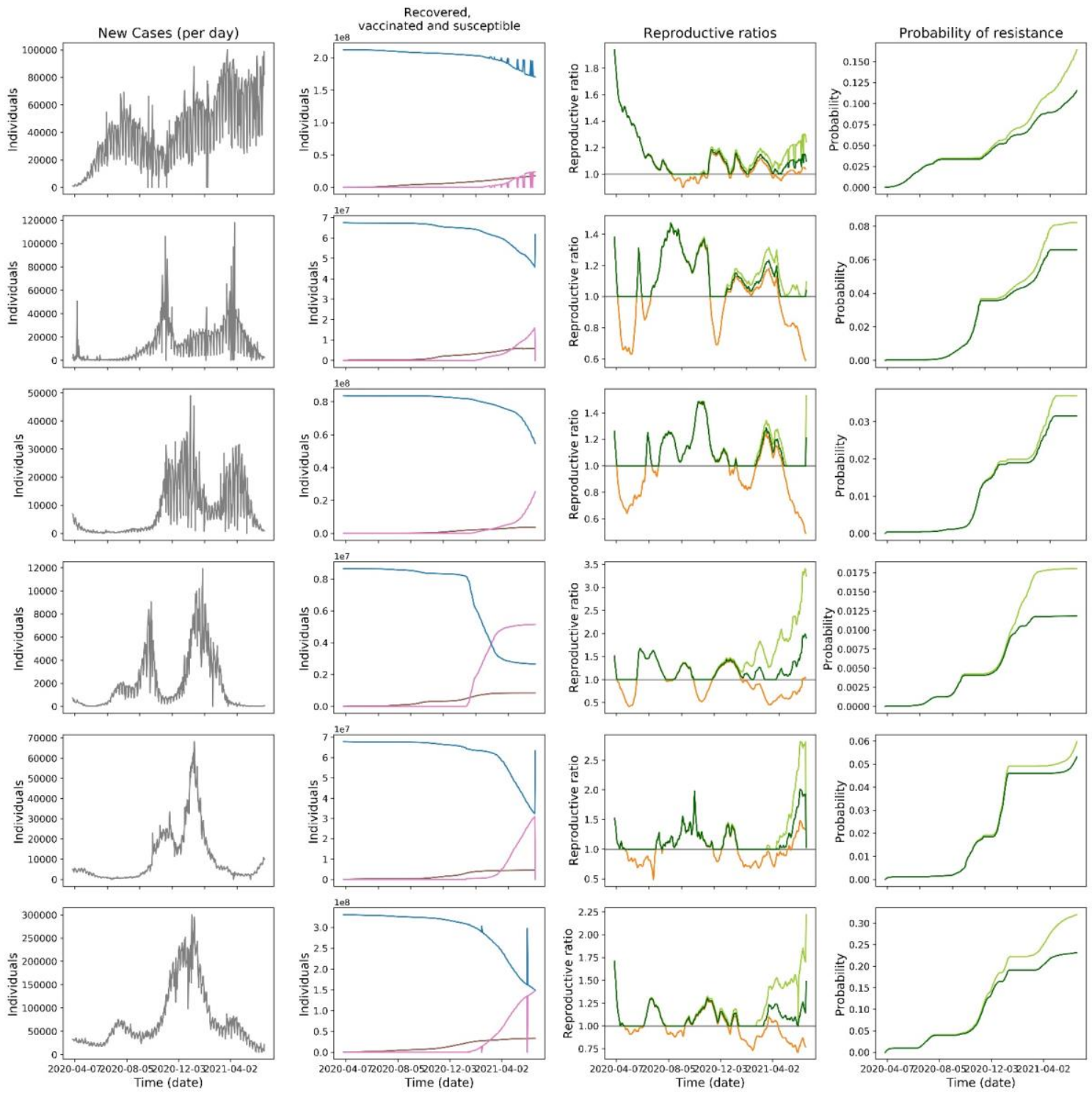

Figure 5: Infection, vaccination data and estimates for reproductive ratios and probability of resistance

for Brazil, France, Germany, Israel, the United Kingdom, and the United States. The total number of new cases per day (leftmost column), number of susceptible,, recovered, and vaccinated individuals (second column from left) was downloaded from the OWID (Our World In Data) database. We used the OWID estimate for the WT reproductive coefficient $R_{W T}$ to calculate the potential MT reproductive coefficient $R_{M T}$ for a full escape mutant ( $q=1$ ) and a partial escape mutant ( $q=0.4$ ), (third column). We use Eq. 2 to estimate the probability that an escape mutant would have emerged until a certain date assuming $\mu=$ $10^{-7}$ (fourth column). 


\begin{tabular}{|c|c|c|c|c|c|}
\hline Country & $\begin{array}{l}\text { Population } \\
\text { size } \\
\left(\times 10^{6}\right)\end{array}$ & $\begin{array}{c}\text { Average } \\
\text { rate of } \\
\text { infection } \\
\text { per day per } \\
\text { person } \\
\text { before } \\
\text { vaccination } \\
\left(\times 10^{-6}\right)\end{array}$ & $\begin{array}{l}\text { Average rate } \\
\text { of infection } \\
\text { per day per } \\
\text { person after } \\
\text { vaccination } \\
\left(\times 10^{-6}\right)\end{array}$ & $\begin{array}{c}\text { Average rate } \\
\text { of } \\
\text { vaccination } \\
\text { per day per } \\
\text { person } \\
\left(\times 10^{-6}\right)\end{array}$ & $\begin{array}{l}\text { Probability of } \\
\text { resistance } \\
\left(\mu=10^{-7}\right)\end{array}$ \\
\hline Brazil & 212.6 & 132 & 294 & 2946 & 0.166 \\
\hline France & 68.1 & 120 & 319 & 4440 & 0.082 \\
\hline Germany & 83.8 & 67 & 135 & 4878 & 0.037 \\
\hline Israel & 8.7 & 120 & 412 & 9181 & 0.018 \\
\hline $\begin{array}{c}\text { United } \\
\text { Kingdom }\end{array}$ & 67.9 & 109 & 187 & 6358 & 0.060 \\
\hline $\begin{array}{l}\text { United } \\
\text { States }\end{array}$ & 331.0 & 173 & 246 & 5533 & 0.320 \\
\hline
\end{tabular}

291 Table 1: Calculated probability of emergence of vaccine resistance using real-world data from six 292 countries: Brazil, France, Germany, Israel, the United Kingdom and the United States. The probability of 293 vaccine resistance was calculated using the product formula in Eq. 2 and the data presented in Figure 5 294 assuming $\mu=10^{-7}$. 


\section{ESTIMATING THE MUTATION RATE $\mu$}

297 We suggest a method to estimate an upper bound of the mutation rate $\mu$ from WT to MT

298 based on the observation that despite a large number of infections since the beginning of 299 the pandemic and including recent vaccination campaigns, no immune evasive mutant 300 has yet taken over. Our method for calculating an upper bound of the mutation rate is 301 potentially applicable for estimating any mutation rate between two phenotypes in an 302 evolving population. The upper bound is computed at any given time point, and can be 303 updated and become tighter if in future evasive strain still does not appear.

304 For each country, we use Eq. (2) to compute the probability of takeover using data from 305 the beginning of the pandemic up until June $19^{\text {th }} 2021$ for a wide range of mutation rates. 306 We assume $q=1$, which means the MT strain is fully immune evasive.

307 The resulting function for probability of mutant take over (for a given time point) versus 308 mutation rate has a sigmoidal shape, with its midpoint corresponding to the mutation 309 rate for which it is equally probable that the MT strain would have taken over or not. 310 Plugging in data on number of infections in several countries gives an upper bound on the 311 probability of mutant take over and a corresponding estimation of mutation rate per 312 transmission that will result in the emergence of such a mutant. This estimated upper 313 bound on the mutation rate decreases over time as long as more infections do not give 314 rise to a MT strain (see Figure 6). 
(A)

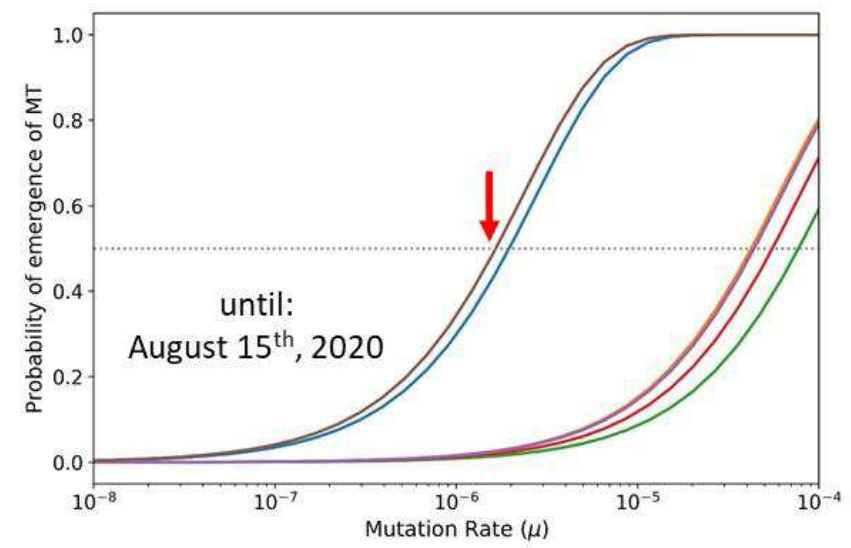

(B)

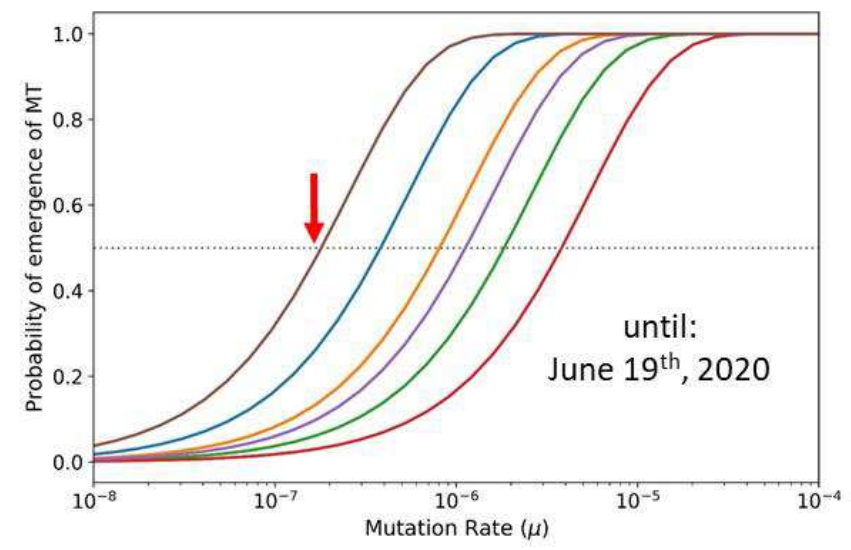

(C)

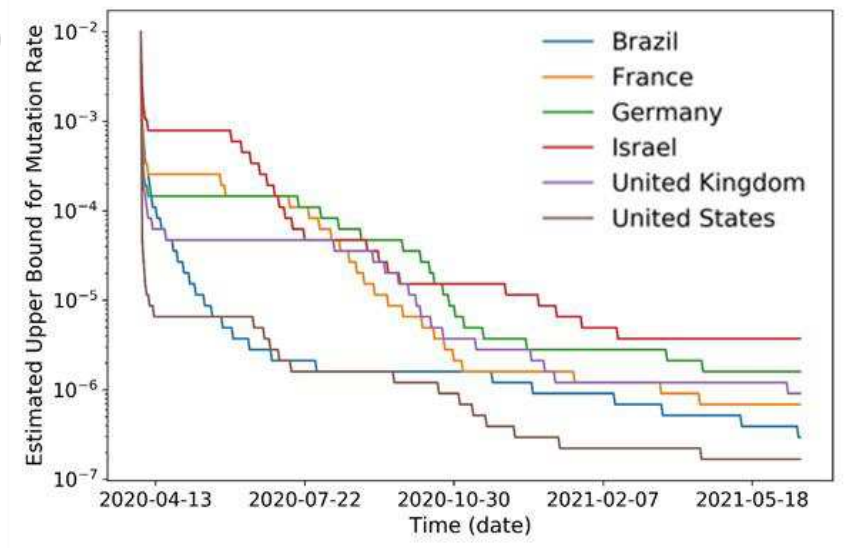

316 Figure 6: Estimation of mutation rate given that no vaccine resistant mutant has yet taken over. $(A, B)$

317 Using Eq. 2, we calculate the probability of MT strain takeover for a range of mutation rate values. The 318 probability of MT strain takeover follows a sigmoidal function, where the midpoint is reached for the value 319 of $\mu$ where MT strain takeover becomes more probable than not. (C) The midpoint of the function 320 (indicated by a red arrow) describing the probability of MT strain takeover will decrease in value as more 321 and more time passes without the takeover of an MT strain. We can use this value as an upper bound of 322 the mutation rate for our model. 
324 Since the probability of MT takeover (Eq. (2)) is strongly dependent on the number of 325 infections, significant decreases in the estimated values correspond to periods with high 326 infection rates in which, nonetheless, a mutant did not appear. The estimate for the upper 327 bound of the mutation rate is expected to plateau as vaccination campaigns lead to a 328 decrease in the number of infection cases. The estimate of $10^{-6}$ will decrease further if 329 and when large countries such as the US will advance in the vaccination campaign with 330 no mutant takeover. Using the world infection and vaccination data, we obtain $\mu=10^{-7}$ 331 as the order of magnitude for the upper bound for the rate at which immune evasive 332 mutants appear. But estimates based on individual countries may be more informative 333 since the world data reflects the average over an extremely heterogeneous population 334 subject to very different policies.

\section{A SIMPLE FORMULA FOR THE ESCAPE PROBABILITY}

336 The dynamic lockdown captured by the social activity parameter $s(t)$ maintains the 337 number of new infections per day fluctuating around a fixed value and thereby buffers 338 the reproductive ratio of the wildtype $R_{W T}$ around 1. The number of active infections is 339 roughly constant and given by $L / a$, where $a$ is the recovery rate (see Figure 3 ). If 340 vaccination is slow, $c \ll L$, then the change in the number of susceptible, $x(t)$, and 341 recovered individuals, $z_{1}(t)$, over time can be described by linear functions with slopes 342 proportional to $L$ (see Methods and Figure S2A).

343 Alternatively, for fast vaccination, $c \gg L$, the change in the number of susceptible $x(t)$

344 and vaccinated individuals $w_{1}(t)$ can be described by linear functions with slopes 345 proportional to $c$ before MT takeover, and with slopes proportional to $L$ after MT 346 takeover (see Methods and Figure 3E). Neglecting vaccination of recovered individuals 347 (which is a reasonable approximation for $c \gg L$ ) we can write $(t)=N-L t-c t, z(t)=$ $348 L t$ and $w(t)=c t$. The time when herd immunity against the WT is reached is given by 


$$
T_{H}=\frac{N}{c+L}\left(1-\frac{1}{R_{0}}\right)
$$

349 During vaccination the reproductive rate of the mutant increases as (see Methods)

$$
R_{M T}(t)=\frac{N}{N-(L+c) t}
$$

350 The reproductive rate of the $\mathrm{MT}$ is initially 1 and increases to $R_{0}$ as people recover from 351 WT infection or are vaccinated (see Figure 3D). Once a mutant has been generated, the 352 probability of its survival depends on the value of the reproductive rate, $R_{M T}(t)$. The 353 probability that no surviving mutant has appeared before time $t$, where $t \leq T_{H}$, can be 354 calculated to be (see Methods):

$$
P(t)=\exp \left[-\left(\frac{\mu N}{2}\right)\left(\frac{L}{N}\right)\left(\frac{c+L}{N}\right) t^{2}\right]
$$

The probability that no surviving mutant has appeared until herd immunity is

$$
P\left(T_{H}\right)=\exp \left[-\left(\frac{\mu N}{2}\right)\left(\frac{L}{c+L}\right)\left(1-\frac{1}{R_{0}}\right)^{2}\right]
$$

Here $R_{0}=\beta N / a$ is the basic reproductive ratio of the WT. The corresponding formulas for partial immune escape mutants are given in the Methods. Eq. (6) is in good agreement with the results of exact stochastic simulations (Figure S3).

In Table 2, we show how the probability and timing of resistance depends on the 360 vaccination rate and the number of new infections per day. We first consider a large country of $N=10^{8}$ inhabitants and a mutation rate of $\mu=10^{-7}$. If 10,000 new infections occur per day and 1 million people are vaccinated per day, then herd immunity is reached in 66 days and the probability of generating a vaccine resistant mutant is about 2 percent. For the same vaccination rate, if 50,000 new infections are tolerated each day, then the probability of generating an escape mutant increases to 10 percent. If 10,000 new infections occur per day but only 100,000 people are vaccinated every day, then the probability of generating vaccine resistance increases to 18 percent. 


\begin{tabular}{|c|c|c|c|c|c|c|}
\hline \multirow{2}{*}{$\begin{array}{l}\text { Rate of } \\
\text { infection } \\
l \text { per day } \\
\text { per person } \\
\left(\times 10^{-6}\right)\end{array}$} & \multirow{2}{*}{$\begin{array}{c}\text { Rate of } \\
\text { vaccination } \\
c \text { per day per } \\
\text { person } \\
\left(\times 10^{-6}\right)\end{array}$} & \multirow{2}{*}{$\begin{array}{l}\text { Time to herd } \\
\text { immunity, } T_{H} \\
\text { (in days) }\end{array}$} & \multicolumn{4}{|c|}{ Probability of resistance } \\
\hline & & & $\begin{array}{l}t=50 \\
\text { days }\end{array}$ & $\begin{array}{c}\mathrm{t}=100 \\
\text { days }\end{array}$ & $\begin{array}{c}\mathrm{t}=200 \\
\text { days }\end{array}$ & $\mathrm{t}=T_{H}$ \\
\hline 100 & 1000 & 606 & 0.001 & 0.005 & 0.022 & 0.183 \\
\hline 100 & 5000 & 131 & 0.006 & 0.025 & - & 0.043 \\
\hline 200 & 5000 & 128 & 0.013 & 0.051 & - & 0.082 \\
\hline 500 & 5000 & 121 & 0.034 & 0.128 & - & 0.183 \\
\hline 100 & 10,000 & 66 & 0.013 & - & - & 0.022 \\
\hline 200 & 10,000 & 65 & 0.025 & - & - & 0.043 \\
\hline 500 & 10,000 & 63 & 0.064 & - & - & 0.100 \\
\hline
\end{tabular}

\begin{tabular}{|c|c|c|c|c|c|c|}
\hline \multirow{2}{*}{$\begin{array}{l}\text { Rate of } \\
\text { infection } \\
l \text { per day } \\
\text { per person } \\
\left(\times 10^{-6}\right)\end{array}$} & \multirow{2}{*}{$\begin{array}{c}\text { Rate of } \\
\text { vaccination } \\
c \text { per day per } \\
\text { person } \\
\left(\times 10^{-6}\right)\end{array}$} & \multirow{2}{*}{$\begin{array}{l}\text { Time to herd } \\
\text { immunity, } T_{H} \\
\text { (in days) }\end{array}$} & \multicolumn{4}{|c|}{ Probability of resistance } \\
\hline & & & $\begin{array}{l}\mathrm{t}=50 \\
\text { days }\end{array}$ & $\begin{array}{c}\mathrm{t}=100 \\
\text { days }\end{array}$ & $\begin{array}{c}t=200 \\
\text { days }\end{array}$ & $\mathrm{t}=T_{H}$ \\
\hline 100 & 1000 & 606 & 0.013 & 0.053 & 0.197 & 0.867 \\
\hline 100 & 5000 & 131 & 0.062 & 0.225 & - & 0.353 \\
\hline 200 & 5000 & 128 & 0.122 & 0.405 & - & 0.575 \\
\hline 500 & 5000 & 121 & 0.291 & 0.747 & - & 0.867 \\
\hline 100 & 10,000 & 66 & 0.119 & - & - & 0.197 \\
\hline 200 & 10,000 & 65 & 0.225 & - & - & 0.353 \\
\hline 500 & 10,000 & 63 & 0.481 & - & - & 0.653 \\
\hline
\end{tabular}

370 Table 2: Calculated probability of vaccine resistance for a range of vaccination rates and infection rates.

371 We observe of counterintuitive effect of higher probability of resistance along time for higher vaccination

372 rates, but lower probability of resistance overall. See also Figure S4. 
375 As the proportion of vaccinated individuals grows, social distancing measures relax, and 376 the probability of emergence of resistance increases. Hence, higher vaccination rates are 377 associated with higher probabilities of resistance after 50, 100 and 200 days (see Table 378 2). However, faster vaccination leads to earlier herd immunity. When herd immunity is 379 reached, there are no more new infections and the cumulative probability of resistance 380 plateaus. Therefore, we observe an interesting counterintuitive effect: the probability of 381 resistance until a fixed time $t$ increases with the vaccination rate $c$, but the probability of 382 resistance until time $T_{H}$ when herd immunity is achieved decreases with the vaccination 383 rate $c$. (see Table 2 and Figure S4).

We can derive estimates for the emergence of vaccine resistant strains using current vaccination and infection rates from around the world. If the whole world $\left(N=8 \cdot 10^{9}\right)$ vaccinated as fast as the US ( $c=5000$ per day per million) and had slightly lower infection rates than Germany ( $L=100$ per day per million) then herd immunity would be achieved in $T_{H}=131$ days; the probability that a resistant virus was generated and survived by that time would be 0.97 (for $\mu=10^{-7}$ ) and 0.29 (for $\mu=10^{-8}$ ). If the whole world vaccinated as fast as Brazil ( $c=3000$ per day per million) and had infection rates like the US ( $L=250$ per day per million) then herd immunity would be achieved in $T_{H}=$ 392205 days; the probability that a resistant virus was generated and survived by that time 393 would be 0.999 (for $\mu=10^{-7}$ ) and 0.75 (for $\mu=10^{-8}$ ). Our results underline the 394 importance of maintaining lockdown measures while herd immunity is not achieved and timely distribution of vaccines around the world.

397 We have studied evolution of resistance to COVID-19 vaccination in the presence of 398 dynamic lockdown. We use real world data to simulate the spread of the SARS-COV-2 399 virus. We have performed stochastic simulations and obtained analytical results. In 400 particular, we have derived a simple intuitive formula for the probability of emergence of 401 a vaccine resistant strain over time (Eqs. (5) and (6)). 
402 Our model most closely corresponds to the assumption that immune evasion could be

403 due to a single point mutation. Nevertheless, our estimates of the mutation rate between

404 the wild-type and immune evasive strains could signify that a combination of mutations

405 is needed to achieve immune evasion. Therefore, we have explored lower effective

406 mutation rates than the current estimation for the per-base mutation rate of the SARS-

407 COV-2 virus.

408 The probability of takeover of an immune evasive strain is mostly dependent on the 409 number of total infection cases that occur during the pandemic. Social distancing 410 measures, such as lockdowns, can delay or event prevent the emergence of the MT strain.

411 Each natural infection is an opportunity for the MT strain to appear and possibly take 412 over. Hence, the main policy goal should be to maximize the proportion of the population 413 which will be immunized to the virus through vaccination as opposed to natural infection.

414 In terms of policy implications, our result supports the maintenance of social distancing 415 measures until the daily number of infections decreases substantially. Allowing a large 416 number of infections can only be counterbalanced by very high vaccination rates, which 417 ensure that herd immunity is reached before the MT strain can appear and takeover. 418 Furthermore, our result underlines the importance of a worldwide effort to quickly 419 vaccinate as many individuals as possible, especially in highly populated countries with 420 low access to vaccines. Slow, or no vaccination, results in a large number of total cases in 421 these areas and hence the emergence of an MT strain which could then spread over the 422 whole world.

\section{ACKNOWLEDGEMENTS}

424 YP acknowledges the Minerva Foundation and Kimmel Foundation for grant support.

\section{AUTHOR CONTRIBUTIONS}

426 All authors conceived the project and designed the study. GL have written all the code and ran 427 analysis. All authors have written the paper. MN has supervised the project.

\section{CODE AND DATA AVAILABILITY}

429 Available upon request. 


\section{REFERENCES}

431 1. Wu, J. T. et al. Estimating clinical severity of COVID-19 from the transmission dynamics in Wuhan, China. Nat. Med. (2020). doi:10.1038/s41591-020-0822-7

$4332 . \quad$ COVID-19 Data Explorer - Our World in Data. Available at:

3. Different COVID-19 Vaccines | CDC. Available at: https://www.cdc.gov/coronavirus/2019-ncov/vaccines/different-vaccines.html. (Accessed: 19th March 2021)

4. Safe COVID-19 vaccines for Europeans | European Commission. Available at: https://ec.europa.eu/info/live-work-travel-eu/coronavirus-response/safe-covid19-vaccines-europeans_en. (Accessed: 19th March 2021)

5. Böttcher, L. \& Nagler, J. Decisive Conditions for Strategic Vaccination against SARS-CoV-2. medRxiv 2021.03.05.21252962 (2021). doi:10.1101/2021.03.05.21252962

6. Walsh, E. E., Shin, J. H. \& Falsey, A. R. Clinical impact of human coronaviruses 229E and OC43 infection in diverse adult populations. J. Infect. Dis. (2013). doi:10.1093/infdis/jit393

7. Vaccines - COVID19 Vaccine Tracker. Available at: https://covid19.trackvaccines.org/vaccines/. (Accessed: 19th March 2021)

454 8. Tegally, H. et al. Emergence and rapid spread of a new severe acute respiratory syndrome-related coronavirus 2 (SARS-CoV-2) lineage with multiple spike mutations in South Africa. medRxiv 10, 2020.12.21.20248640 (2020). 
457 9. Rambaut, A. et al. Preliminary genomic characterisation of an emergent SARSCoV-2 lineage in the UK defined by a novel set of spike mutations - SARS-CoV-2 coronavirus / nCoV-2019 Genomic Epidemiology - Virological. (2020). Available at: https://virological.org/t/preliminary-genomic-characterisation-of-an-emergentsars-cov-2-lineage-in-the-uk-defined-by-a-novel-set-of-spike-mutations/563. (Accessed: 16th April 2021)

10. Wang, P. et al. Increased Resistance of SARS-CoV-2 Variants B.1.351 and B.1.1.7 to Antibody Neutralization. bioRxiv Prepr. Serv. Biol. (2021). doi:10.1101/2021.01.25.428137

11. Luchsinger, L. L. \& Hillyer, C. D. Vaccine efficacy probable against COVID-19 variants. Science (80-. ). 371, 1116 LP - 1116 (2021).

12. Starr, T. N. et al. Prospective mapping of viral mutations that escape antibodies used to treat COVID-19. Science (80-. ). 371, 850-854 (2021).

13. Starr, T. N. et al. Deep Mutational Scanning of SARS-CoV-2 Receptor Binding Domain Reveals Constraints on Folding and ACE2 Binding. Cell 182, 12951310.e20 (2020).

14. Greaney, A. J. et al. Complete Mapping of Mutations to the SARS-CoV-2 Spike Receptor-Binding Domain that Escape Antibody Recognition. Cell Host Microbe 29, 44-57.e9 (2021).

15. Thompson, R. N., Hill, E. M. \& Gog, J. R. SARS-CoV-2 incidence and vaccine escape. Lancet Infect. Dis. (2021). doi:10.1016/S1473-3099(21)00202-4

16. Gerrish, P. J. et al. How unequal vaccine distribution promotes the evolution of vaccine escape. medRxiv (2021).

17. Cobey, S., Larremore, D. B., Grad, Y. H. \& Lipsitch, M. Concerns about SARS-CoV-2 evolution should not hold back efforts to expand vaccination. Nature Reviews Immunology 1-6 (2021). doi:10.1038/s41577-021-00544-9 
18. Geoffroy, F., Traulsen, A. \& Uecker, H. Vaccination strategies when vaccines are scarce: On conflicts between reducing the burden and avoiding the evolution of escape mutants. medRxiv 2021.05.04.21256623 (2021). doi:10.1101/2021.05.04.21256623

19. Baker, R. E., Yang, W., Vecchi, G. A., Metcalf, C. J. E. \& Grenfell, B. T. Assessing the influence of climate on wintertime SARS-CoV-2 outbreaks. Nat. Commun. 12, 1-7 (2021).

20. Geoghegan, J. L. et al. Genomic epidemiology reveals transmission patterns and dynamics of SARS-CoV-2 in Aotearoa New Zealand. Nat. Commun. 11, 1-7 (2020).

21. Komissarov, A. B. et al. Genomic epidemiology of the early stages of the SARSCoV-2 outbreak in Russia. Nat. Commun. 12, 1-13 (2021).

22. Faria, N. R. et al. Genomics and epidemiology of the P.1 SARS-CoV-2 lineage in Manaus, Brazil. Science (80-. ). eabh2644 (2021). doi:10.1126/science.abh2644

23. Lythgoe, K. A. et al. SARS-CoV-2 within-host diversity and transmission. Science (80-. ). 372, eabg0821 (2021).

24. Komarova, N. L., Schang, L. M. \& Wodarz, D. Patterns of the COVID-19 pandemic spread around the world: Exponential versus power laws: Patterns of the COVID19 pandemic spread around the world: Exponential versus power laws. J. R. Soc. Interface 17, (2020).

25. Saad-Roy, C. M. et al. Immune life history, vaccination, and the dynamics of SARSCoV-2 over the next 5 years. Science (80-. ). 370, 811-818 (2020).

26. BERNOULLI \& D. Essai d'une nouvelle analyse de la mortalite causee par la petite verole, et des avantages de l'inoculation pour la prevenir. Hist. l'Acad., Roy. Sci. avec Mem 1-45 (1760).

27. Kermack, W. 0 \& Mckendrick, A. G. A contribution to the mathematical theory of epidemics. Proc. R. Soc. London. Ser. A, Contain. Pap. a Math. Phys. Character 
115, 700-721 (1927).

28. Diekmann, O., Heesterbeek, J. A. P. \& Metz, J. A. J. On the definition and the computation of the basic reproduction ratio RO in models for infectious diseases in heterogeneous populations. J. Math. Biol. 28, 365-382 (1990).

29. Dietz, K. The estimation of the basic reproduction number for infectious diseases. Stat. Methods Med. Res. 2, 23-41 (1993).

30. Nowak, M. A. \& May, R. M. Superinfection and the evolution of parasite virulence. Proc. R. Soc. B Biol. Sci. 255, 81-89 (1994).

31. Nowak, M. A. \& Bangham, C. R. M. Population dynamics of immune responses to persistent viruses. Science (80-. ). 272, 74-79 (1996).

32. Hethcote, H. W. Mathematics of infectious diseases. SIAM Rev. 42, 599-653 (2000).

33. Brauer, F. \& Castillo-Chavez, C. Mathematical Models in Population Biology and Epidemiology. 40, (Springer New York, 2012).

34. Hamer, W. The Milroy Lectures ON EPIDEMIC DISEASE IN ENGLAND-THE EVIDENCE OF VARIABILITY AND OF PERSISTENCY OF TYPE. The Lancet 167, 569574 (1906).

35. Bailey, N. The Mathematical Theory of Infectious Diseases and its applications. Immunology 34, (Wiley-Blackwell, 1978).

36. Tillett, H. E. Infectious Diseases of Humans; Dynamics and Control. Epidemiol. Infect. 108, 211 (1992).

37. Nowak, M. \& May, R. M. Virus Dynamics: Mathematical Principles of Immunology And Virology. Available at: https://www.researchgate.net/publication/48378467_Virus_Dynamics_Mathema tical_Principles_of_Immunology_And_Virology. (Accessed: 30th March 2021) 
38. Diekmann, O. \& Heesterbeek, J. A. P. Mathematical Epidemiology of Infectious Diseases: Model Building, Analysis and Interpretation. Available at: https://www.researchgate.net/publication/48376881_Mathematical_Epidemiolo gy_of_Infectious_Diseases_Model_Building_Analysis_and_Interpretation. (Accessed: 30th March 2021)

39. Ragonnet-Cronin, M. et al. Genetic evidence for the association between COVID19 epidemic severity and timing of non-pharmaceutical interventions. Nat. Commun. 12, 2188 (2021).

40. Rossman, H. et al. Hospital load and increased COVID-19 related mortality in Israel. Nat. Commun. 12, 1904 (2021).

41. Saad-Roy, C. M. et al. Epidemiological and evolutionary considerations of SARSCoV-2 vaccine dosing regimes. Science (80-. ). eabg8663 (2021). doi:10.1126/science.abg8663

42. Saad-Roy, C. M. et al. Immune life history, vaccination, and the dynamics of SARSCoV-2 over the next 5 years. Science (80-. ). 370, 811-818 (2020).

43. Ashcroft, P., Lehtinen, S., Angst, D. C., Low, N. \& Bonhoeffer, S. Quantifying the impact of quarantine duration on covid-19 transmission. Elife 10, 1-33 (2021).

44. Komarova, N. L., Azizi, A. \& Wodarz, D. Network models and the interpretation of prolonged infection plateaus in the COVID19 pandemic. Epidemics 100463 (2021). doi:10.1016/j.epidem.2021.100463

45. Castro, M., Ares, S., Cuesta, J. A. \& Manrubia, S. The turning point and end of an expanding epidemic cannot be precisely forecast. Proc. Natl. Acad. Sci. 117, 26190-26196 (2020).

46. Stich, M., Manrubia, S. C. \& Lázaro, E. Variable mutation rates as an adaptive strategy in replicator populations. PLoS One (2010). doi:10.1371/journal.pone.0011186 
47. Yagan, O. et al. Modeling and Analysis of the Spread of COVID-19 Under a Multiple-Strain Model with Mutations. Harvard Data Sci. Rev. (2021). doi:10.1162/99608f92.a11bf693

48. Lehtinen, S., Ashcroft, P. \& Bonhoeffer, S. On the relationship between serial interval, infectiousness profile and generation time. J. R. Soc. Interface (2021). doi:10.1098/rsif.2020.0756

49. Hethcote, H. W. Three Basic Epidemiological Models. in (1989). doi:10.1007/9783-642-61317-3_5

50. Gillespie, D. T. A general method for numerically simulating the stochastic time evolution of coupled chemical reactions. J. Comput. Phys. 22, 403-434 (1976).

51. Gillespie, D. T. Exact stochastic simulation of coupled chemical reactions. in Journal of Physical Chemistry 81, 2340-2361 (American Chemical Society, 1977).

52. Cota, W. \& Ferreira, S. C. Optimized Gillespie algorithms for the simulation of Markovian epidemic processes on large and heterogeneous networks. Comput. Phys. Commun. 219, 303-312 (2017).

53. Mukhamadiarov, R. I. et al. Social distancing and epidemic resurgence in agentbased susceptible-infectious-recovered models. Sci. Rep. 11, 130 (2021).

8 METHODS

9 Derivation of mathematical results

1. No Vaccination

881 First we consider the case without vaccination. We denote by $x$ the number of susceptible 82 individuals; by $y$ the number of individuals infected with wildtype (WT); by $z$ the number 883 of individuals recovered from WT. The infection rate is $\beta$; the recovery rate $a$; the 884 mutation rate $\mu$; the population size $N$. The social activity parameter $s(t)$ captures the 
extent of imposed lockdown that varies over time. For simplicity, we neglect the number

586 of individuals who die, hence the population size $N$ is assumed to be constant.

587 Deterministic WT infection dynamics are given by the system of differential equations:

$$
\begin{gathered}
\dot{x}=-\beta s x y \\
\dot{y}=\beta s x y-a y \\
\dot{z}=a y
\end{gathered}
$$

588 Initially, all of the population is susceptible to the WT strain, and no individuals are 589 infected with or recovered from the WT strain. Therefore, we have: $x(0)=N, y(0)=0$ 590 and $z(0)=0$. Social activity, $s(t)$, is adjusted such that $y(t)=L / a$ is constant (see 591 Figure S2C). $L$ is the number of new infections per day.

592 Without lockdown, $s=1$, the basic reproductive ratio of the WT is given by $R_{0}=\beta N / a$. 593 If $R_{0}>1$, the number of infected individuals grows initially. With lockdown, $s<1$, the 594 reproductive ratio is $R_{W T}=\beta N s / a$. Since the social distancing measures maintain $595 y(t)=L / a$ at a constant value, we have $R_{W T}=1$ and $\beta s(t) x(t)=a$ (see Figure S2). 596 The parameter $s$ can vary between 0 and 1.

597 Each day, $L$ individuals become infected and $L$ individuals recover. Therefore, we have:

$$
\begin{gathered}
\dot{x}=-L \\
\dot{y}=0 \\
\dot{z}=L
\end{gathered}
$$

598 The solution to this system of differential equations is

$$
\begin{gathered}
x(t)=N-L t \\
z(t)=L t
\end{gathered}
$$

599 Hence, the number of susceptible individuals decreases linearly with slope $L$ while the 600 number of recovered individuals increases linearly with slope $L$. (See Figure S2 for 601 agreement with the stochastic simulation). 
602 When $x(t)$ has declined such that $R_{W T}<1$ and $s=1$, there are not enough susceptible 603 individuals to sustain the infection. This herd immunity is achieved when $x(t)<a / \beta$. 604 Thus, the time $T_{H}$ until herd immunity is given by $\beta(N-L t)=a$. We obtain:

$$
T_{H}=\frac{N}{L}\left(1-\frac{1}{R_{0}}\right)
$$

\section{$605 \quad 1.1$ Rate of generating mutants}

606 Each day, $L$ new individuals become infected. Each of these infections has probability $\mu$ 607 to be a vaccine-resistant MT. Hence, the rate of producing a mutant is $L \mu$ per day. Let $608 P(t)$ denote the probability that no mutant has been produced until time $t$. We have $609 P \dot{P}(t)=-L \mu P(t)$, which leads to $P(t)=e^{-L \mu t}$.

610 The MT strain can be generated only during infection. Hence, if the MT strain has not 611 been generated until the time when there are no more WT infections - that is, 612 approximately when herd immunity is reached - it will never be generated. We neglect 613 here the time of exponential decrease in the number of WT infections between time $T_{H}$ 614 (when herd immunity is reached) and the time when the number of WT infections has 615 reached zero. The probability that no mutant will appear before time $T_{H}$ is $P\left(T_{H}\right)=$ $616 e^{-L \mu T_{H}}$. Inserting from eq (4) we obtain

$$
P\left(T_{H}\right)=\exp \left(-N \mu\left(1-1 / R_{0}\right)\right.
$$

\section{$617 \quad 1.1$ Rate of generating surviving mutants}

618 In order to calculate the probability that the MT strain will be generated and survive, we 619 need to multiply the rate of generation of the MT strain with the probability that it will 620 not become extinct by random drift. If $\rho(t)$ is the survival probability of the MT, then the 621 rate of producing a surviving mutant is $L \mu \rho(t)$ per day. We approximate $\rho(t)=1-$ $6221 / R_{M T}(t)$, where $R_{M T}(t)$ is the reproductive ratio of the mutant at time $T$. 


$$
R_{M T}(t)=\beta s(t) N / a
$$

624 Since $s(t)=a / \beta x(t)$ and using Eq. (9) we obtain

$$
R_{M T}(t)=\frac{N}{N-L t}
$$

625 And therefore we have $\rho(t)=L t / N$.

626 Let $P(t)$ denote the probability that not surviving mutant has been produced until time

$627 t$. We have $P \dot{(t)}=-L \mu \rho(t) P(t)=-L^{2} \mu t P(t) / N$. We can solve this differential

628 equation to obtain $P(t)=\exp \left(-\mu L^{2} t^{2} / 2 N\right)$. The probability that no surviving mutant

629 has been produced until herd immunity, which is reached at time $T_{H}$, is given by

$$
P\left(T_{H}\right)=\exp \left(-\frac{\mu N}{2}\left(1-\frac{1}{R_{0}}\right)^{2}\right)
$$

\section{2. With Vaccination}

631 Let us now add vaccination. Denote by $w$ the number of vaccinated people. If both

632 recovered and susceptible individuals are vaccinated at a total rate of $c$ per day then

633 deterministic infection and vaccination dynamics are given by

$$
\begin{gathered}
\dot{x}=-\beta s x y-\frac{c x}{x+z} \\
\dot{y}=\beta s x y-a y \\
\dot{z}=a y-\frac{c z}{x+z} \\
\dot{w}=c
\end{gathered}
$$

634 The initial condition is $x(0)=N, y(0)=0, z(0)=0, w(0)=0, s(0)=1$ and $R_{0}=$ $635 \beta N / a$. As before, we adjust $s(t)$ such that $y(t)=L / a$ is constant (see Figure 3 ).

636 Each day, $L$ susceptible individuals become infected and $c x(x+z)$ susceptible individuals 637 become vaccinated. Also, $L$ infected individuals recover, and $c x(x+z)$ of recovered 638 individuals become vaccinated. We have: 


$$
\begin{gathered}
\dot{x}=-L-\frac{c x}{x+z} \\
\dot{y}=0 \\
\dot{z}=L-\frac{c z}{x+z} \\
\dot{w}=c
\end{gathered}
$$

639 For simplicity let us assume that we only vaccinate susceptible people. This assumption 640 is a reasonable approximation if $c \gg L$. In this case, we can write

$$
\begin{gathered}
\dot{x}=-L-c \\
\dot{y}=0 \\
\dot{z}=L \\
\dot{w}=c
\end{gathered}
$$

642 The solution to this system of differential equations is

$$
\begin{gathered}
x(t)=N-L t-c t \\
z(t)=L t \\
w(t)=c t
\end{gathered}
$$

643 Hence, the number of susceptible individuals decreases linearly with slope $L+c$, while 644 the number of recovered individuals increases linearly with slope $L$, and the number of 645 vaccinated individuals increases linearly with slope $c$.

646 The time $T_{H}$ until herd immunity is given by

$$
T_{H}=\frac{N}{c+L}\left(1-1 / R_{0}\right)
$$




\subsection{Rate of generating mutants}

648 The rate of producing a mutant is $L \mu$ per day. Let $P(t)$ denote the probability that no 649 mutant has been produced until time $t$. We have $P \dot{(t)}=-L \mu P(t)$, which gives $P(t)=$ $650 \exp (-L \mu t)$.

651 The MT strain can be generated only during infection. Hence, if the MT strain has not 652 been generated until the time when there are no more WT infections - that is, when herd 653 immunity is reached - it will never be generated. Again we neglect here the time of 654 exponential decrease in the number of WT infections between the time $T_{H}$ when herd 655 immunity is reached and the time where the number of WT infections reaches 0 . Hence, 656 the probability that no mutant will appear is $P\left(T_{H}\right)=\exp \left(-L \mu T_{H}\right)$. Using Eq. (19), the 657 probability that no mutant has appeared until herd immunity is:

$$
P\left(T_{H}\right)=\exp \left(-N \mu\left(\frac{L}{c+L}\right)\left(1-\frac{1}{R_{0}}\right)\right)
$$

\subsection{Rate of generating surviving mutants}

659 In order to calculate the probability that surviving mutants are generated, we again 660 consider the survival probability $\rho(t)=1-1 / R_{M T}(t)$, where $R_{M T}(t)$ is the reproductive 661 ratio of the mutant at time $t$. The rate of producing a surviving mutant is then $L \mu \rho(t)$ per 662 day. We have:

$$
R_{M T}(t)=\frac{\beta s(t) N}{a}
$$

663 As explained above, $s(t)=a / \beta x(t)$. Using Eq. (18) we obtain

$$
R_{M T}(t)=\frac{N}{N-(L+c) t}
$$

664 And therefore $\rho(t)=(L+c) t / N$. 
665 Let $P(t)$ denote the probability that not surviving mutant has been produced until time $666 t$. We have $P \dot{(t)}=-L \mu \rho(t) P(t)=-L \mu(c+L) t P(t) / N$. Let $v=c / N$ and $l=L / N$.

667 We can solve this differential equation to obtain:

$$
P(t)=\exp \left(-\frac{\mu N}{2} l(v+l) t^{2}\right)
$$

668 The probability that no surviving mutant has been produced until herd immunity, which 669 is reached at time $T_{H}$, is:

$$
P\left(T_{H}\right)=\exp \left(-\frac{\mu N}{2}\left(\frac{l}{v+l}\right)\left(1-\frac{1}{R_{0}}\right)^{2}\right)
$$

670

6712.3 Rate of generating surviving mutants with partial immune escape

672 We can also study the case where the infectivity of the mutants is reduced by a factor

$673 q \in[0,1]$ when infecting recovered or vaccinated people. For $q=1$ we obtain full

674 escape, while $q=0$ means that the mutant does not escape at all.

675 A similar derivation to the one above leads to the following result. The probability that

676 no surviving mutant with partial escape $q$ has appeared until herd immunity is given by:

$$
\text { with } \begin{aligned}
P\left(T_{H}\right) & =\exp \left(-\frac{\mu N}{2}\left(\frac{l}{v+l}\right) A\right) \\
\quad A & =\frac{2 q}{1-q}\left(-\frac{R_{0}-1}{R_{0}}+\frac{1}{1-q} \log \frac{R_{0}}{1+q\left(R_{0}-1\right)}\right)
\end{aligned}
$$

677 For $q=1$ we obtain $A=\left(1-\left(1 / R_{0}\right)\right)^{2}$ leading to Eq. (23) above. 
681 Each day, $L$ new WT infections occur. Each new infection has a probability of $\mu$ to be the 682 MT strain. The survival probability of the mutant is approximately $1-1 / R_{m}(t)$ where $683 R_{m}(t)$ is the basic reproductive ratio of the MT appearing at time $t$.

684 Hence, the probability that none of the $L$ new WT infections in a day will generate a 685 surviving mutant is $\left(1-\mu\left(1-1 / R_{m}(t)\right)\right)^{L}$. Then, we can write the probability $P$ that no 686 surviving mutant will be produced between time $t=0$ and the time $T_{H}$ when herd 687 immunity is reached as the product

$$
P=\prod_{\tau=0}^{T_{H}}\left[1-\mu\left(1-\frac{1}{R_{M T}(\tau)}\right)\right]^{L}
$$

We have $T_{H}=[N /(c+L)]\left(1-1 / R_{0}\right)$ and $R_{M T}(t)=N /[N-(c+L) t]$.

Since $\rho(t)=1-1 / R_{m}(t)=(c+L) t / N$ we can write:

$$
P=\prod_{\tau=0}^{T_{H}}\left(1-\frac{\mu(c+L) \tau}{N}\right)^{L}
$$

691 Let us use the abbreviation $u=\mu(c+L) / N$. Then

$$
P=\prod_{\tau=0}^{T_{H}}(1-u \tau)^{L}
$$

$$
=\exp \left[\log \prod_{\tau=0}^{T_{H}}(1-u \tau)^{L}\right]
$$

694

$$
\begin{gathered}
=\exp \left[L \log \prod_{\tau=0}^{T_{H}}(1-u \tau)\right] \\
=\exp \left[L \sum_{\tau=0}^{T_{H}} \log (1-u \tau)\right]
\end{gathered}
$$

695 Note that Eq. (26) is exactly equivalent to Eq. (23). Assuming $u T_{H} \ll 1$ which is the same 696 as $\mu\left(1-\left(1 / R_{0}\right)\right) \ll 1$ we obtain 


$$
\begin{gathered}
P=\exp \left[-u L \sum_{\tau=0}^{T_{H}} \tau\right] \\
=\exp \left[-\frac{u L T_{H}\left(T_{H}+1\right)}{2}\right]
\end{gathered}
$$

Assuming $T_{H} \gg 1$ which is $N\left(1-\frac{1}{R_{0}}\right) \gg c+L$, we obtain

700

$$
\begin{gathered}
P=\exp \left[-\frac{u L T_{H}^{2}}{2}\right] \\
=\exp \left[-\frac{(\mu(c+L) / N) L T_{H}^{2}}{2}\right]
\end{gathered}
$$

702 Finally, inserting $T_{H}=(N /(c+L))\left(1-1 / R_{0}\right)$ we get:

$$
P\left(T_{H}\right)=\exp \left(-\frac{\mu N}{2}\left(\frac{l}{v+l}\right)\left(1-\frac{1}{R_{0}}\right)^{2}\right)
$$

703 Which is equivalent to Eq.(24) (see above).

704

705

\section{Dynamics after appearance of the MT strain}

706 1. No Vaccination

707 After the MT strain has taken over, social distancing measures will continue maintaining 708 the number of daily infections at $L$, which implies that $\left(y_{1}+y_{2}\right)=L / a$ (see Figure S2). 709 In practice, the WT strain rapidly goes extinct upon emergence of the MT strain; so we 710 can consider $y_{2}=L / a$.The mutant strain can infect susceptible individuals $x$, and 711 recovered individuals, $z_{1}$. The mutant strain infects those individuals with probabilities 712 proportional to their frequencies at the time $t^{*}$ of mutant takeover. Hence, for times $t>$ $713 t^{*}$ we have:

$$
\begin{gathered}
x(t)=x\left(t^{*}\right)-\frac{x\left(t^{*}\right)}{z_{1}\left(t^{*}\right)+x\left(t^{*}\right)} L\left(t^{*}-t\right) \\
z_{1}(t)=z_{1}\left(t^{*}\right)-\frac{z_{1}\left(t^{*}\right)}{z_{1}\left(t^{*}\right)+x\left(t^{*}\right)} L\left(t^{*}-t\right)
\end{gathered}
$$


714 After mutant takeover, the social distancing measures need to be readjusted to the 715 mutant strain. Since more individuals are susceptible to it, $s(t)$ has to decrease (see 716 Figure S2F):

$$
s(t)=\frac{a N}{\beta\left(x(t)+q z_{1}(t)\right)}
$$

717 Which implies that $R_{M T}=1$.

718

719

2. With vaccination

720 As for the case without vaccination, if the mutant strain survives, it will quickly replace 721 the wild-type strain such that $y_{2}=L / a$ (see Figure $3 C$ ). The number of susceptible 722 individuals $x\left(t^{*}\right)$ at time of mutant takeover can be neglected for large enough 723 vaccination rates. The number of vaccinated individuals, susceptible to the mutant strain $724 w_{1}$ will hence decrease linearly with the number of tolerated cases per day $L$, and the 725 number of vaccinated individuals, recovered from the mutant strain $w_{2}$ will increase 726 complementarily linearly with $L$. If the mutant takes over at time $t^{*}$, we have for all times $727 t>t^{*}:$

$$
\begin{gathered}
w_{1}(t)=w_{1}\left(t^{*}\right)-L\left(t^{*}-t\right) \\
w_{2}(t)=L\left(t^{*}-t\right)
\end{gathered}
$$

728 The social activity parameter $s$ needs readjustment to consider the additional groups of 729 individuals that are now susceptible to the infecting strain. We have:

$$
s(t)=\frac{a}{\beta} \frac{x(t)+q\left(z_{1}(t)+w_{1}(t)\right.}{x(t)+q\left(z_{1}(t)+w_{1}(t)\right)-w_{2}(t)}
$$

730 Which ensures that $R_{M T}=1$. Here the parameter $q$ in $[0,1]$ denotes the extent of escape. 


\section{Estimating the evolutionary potential of the virus}

733 If $\mu$ is the mutation rate as described above and $L(t)$ is the time series giving the

734 number of new infections on day $t$, then the probability that no mutant has been

735 produced between time 0 and time $T_{H}$ is given by:

$$
P\left(T_{H}\right)=\prod_{\tau=0}^{T_{H}}[1-\mu]^{L(\tau)}
$$

736 This probability will overestimate the evolutionary potential of the virus to escape from 737 vaccination because many mutants do not survive the initial random drift. The probability 738 that no surviving mutant has been produced between time 0 and time $T_{H}$ can be written 739 as:

$$
P\left(T_{H}\right)=\prod_{\tau=0}^{T_{H}}[1-\mu \rho(\tau)]^{L(\tau)}
$$

740 Here $\rho(t)$ is the survival probability of an escape mutant produced at time $t$. This 741 probability depends on the basic reproductive ratio of the mutant on the day it is being 742 produced (and the next few days until random drift is negligible). Approximately we can 743 write:

$$
\rho(t)=\min \left\{0,1-\frac{1}{R_{M}(t)}\right\}
$$

744 For the potential of the virus to generate mutants (irrespective of whether they survive) 745 what matters most is the total number of infections, $\sum_{\tau} L(\tau)$. But for the potential of the 746 virus to generate surviving mutants one must also consider the time periods when 747 lockdown is relaxed such that $R_{M T}$ is above 1 .

$$
\text { Time }=0
$$




$$
\operatorname{day}=\operatorname{day}+1
$$

if number infections in previous day $>$ L: between 0 and 1 between 0 and 1 
778 TABLE OF REACTIONS AND THEIR RATES

779

\begin{tabular}{|c|c|c|}
\hline \multicolumn{3}{|c|}{ Infection } \\
\hline $\begin{array}{l}\text { WT Infected Infects } \\
\text { Susceptible }\end{array}$ & $y_{1}+1 ; x-1$ & $\beta_{1} s x y_{1}$ \\
\hline $\begin{array}{l}\text { VR (unvaccinated) Infects } \\
\text { Susceptible }\end{array}$ & $y_{2 A}+1 ; x-1$ & $\beta_{2} S x y_{2 A}$ \\
\hline $\begin{array}{l}\text { VR (unvaccinated) Infects } \\
\text { Recovered from WT }\end{array}$ & $y_{2 A}+1 ; z_{1}-1$ & $q \beta_{2} S z_{1} y_{2 A}$ \\
\hline $\begin{array}{c}\text { VR (unvaccinated) Infects } \\
\text { Vaccinated }\end{array}$ & $y_{2 B}+1 ; w_{1}-1$ & $q \beta_{2} S w_{1} y_{2 A}$ \\
\hline $\begin{array}{l}\text { VR (vaccinated) Infects } \\
\text { Susceptible }\end{array}$ & $y_{2 A}+1 ; x-1$ & $\beta_{2} S x y_{2 B}$ \\
\hline $\begin{array}{l}\text { VR (vaccinated) Infects } \\
\text { Recovered from WT }\end{array}$ & $y_{2 A}+1 ; z_{1}-1$ & $q \beta_{2} z_{1} s y_{2 B}$ \\
\hline $\begin{array}{l}\text { VR (vaccinated) Infects } \\
\text { Vaccinated }\end{array}$ & $y_{2 B}+1 ; w_{1}-1$ & $q \beta_{2} w_{1} s y_{2 B}$ \\
\hline \multicolumn{3}{|c|}{ Mutation } \\
\hline
\end{tabular}




\begin{tabular}{|c|c|c|}
\hline WT Mutates into VR & $x-1 ; y_{2 A}+1$ & $\beta_{1} s x y_{1} \mu$ \\
\hline \multicolumn{3}{|c|}{$\underline{\text { Recovery }}$} \\
\hline WT Infected Recovers & $y_{1}-1 ; z_{1}+1$ & $a y_{1}$ \\
\hline VR unvaccinated Recovers & $y_{2 A}-1 ; z_{2}+1$ & $a y_{2 A}$ \\
\hline VR vaccinated Recovers & $y_{2 B}-1 ; w_{2}+1$ & $a y_{2 B}$ \\
\hline \multicolumn{3}{|c|}{ Death } \\
\hline WT Infected Dies & $y_{1}-1$ & $d y_{1}$ \\
\hline VR unvaccinated Dies & $y_{2 A}-1$ & $d y_{2 A}$ \\
\hline VR vaccinated Dies & $y_{2 B}-1$ & $d y_{2 B}$ \\
\hline \multicolumn{3}{|c|}{ Vaccination } \\
\hline $\begin{array}{c}\text { Susceptible Gets } \\
\text { Vaccinated }\end{array}$ & $x-1 ; w_{1}+1$ & $\frac{c x}{\left(x+z_{1}+z_{2}\right)}$ \\
\hline $\begin{array}{c}\text { WT Recovered Gets } \\
\text { Vaccinated }\end{array}$ & $z_{1}-1 ; w_{1}+1$ & $\frac{c z_{1}}{\left(x+z_{1}+z_{2}\right)}$ \\
\hline $\begin{array}{c}\text { WT and VR Recovered Gets } \\
\text { Vaccinated }\end{array}$ & $z_{2}-1 ; w_{2}+1$ & $\frac{c z_{2}}{\left(x+z_{1}+z_{2}\right)}$ \\
\hline
\end{tabular}



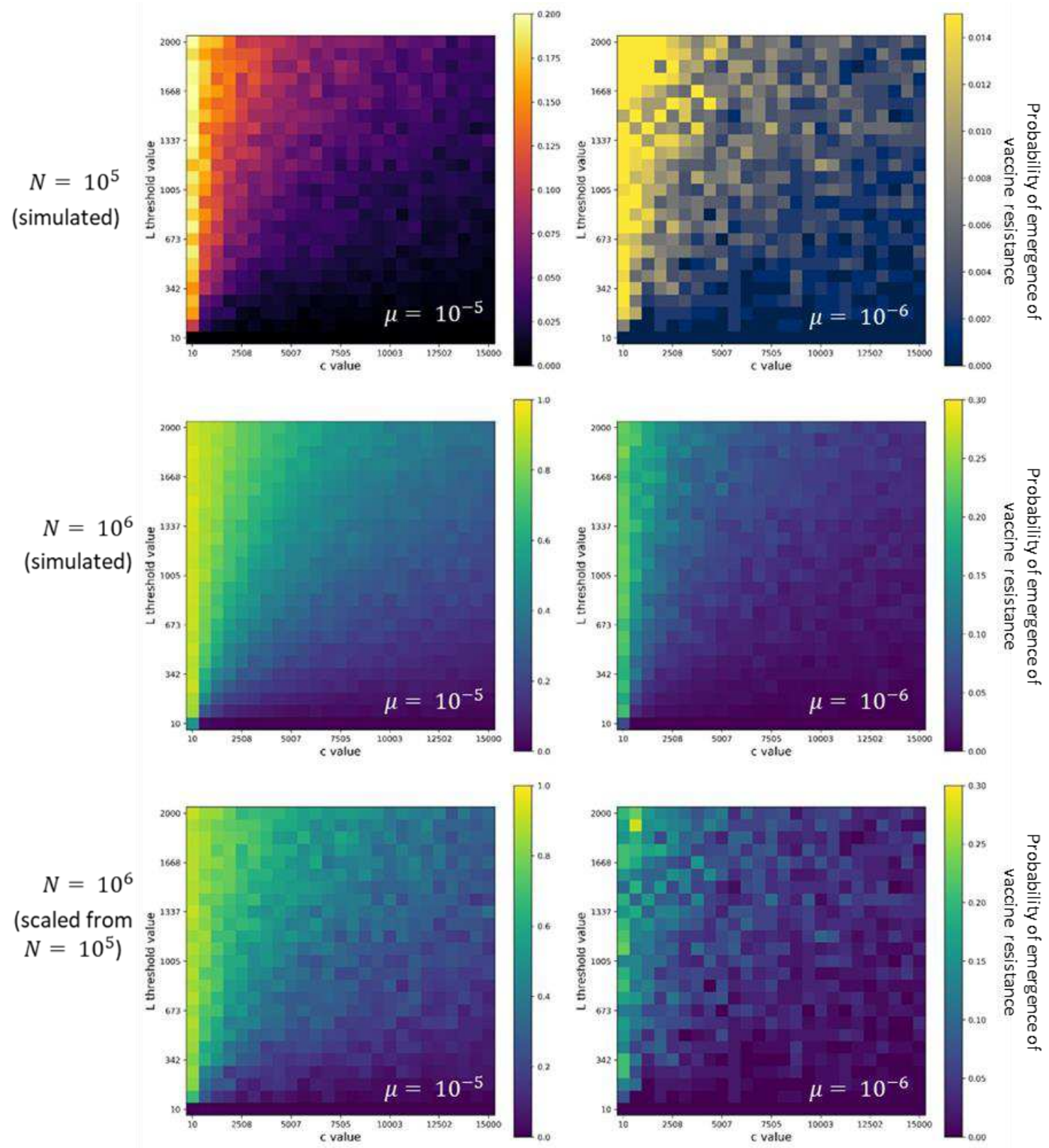

Figure S1: Scaling simulation results to larger population sizes. Results of simulations for a given population size can be scaled to larger population size according to $1-(1-p)^{m}$, where $\mathrm{p}$ is the proportion of runs where the MT strain took over and $m$ the ratio of the scaled population size to the simulated population size. (A) (B) Each square of the color map is colored according to the proportion of runs (out of 100) where the MT strain took over for $N=10^{5}$. (C) (D) Each square of the color map is colored according to 
791 (A) and (B). (E) (F) Each square of the color map is colored according to the proportion of runs (out of 100)

792 where the MT strain took over for $N=10^{6}$. We observe a good agreement between the scaled results and 793 the simulated results.
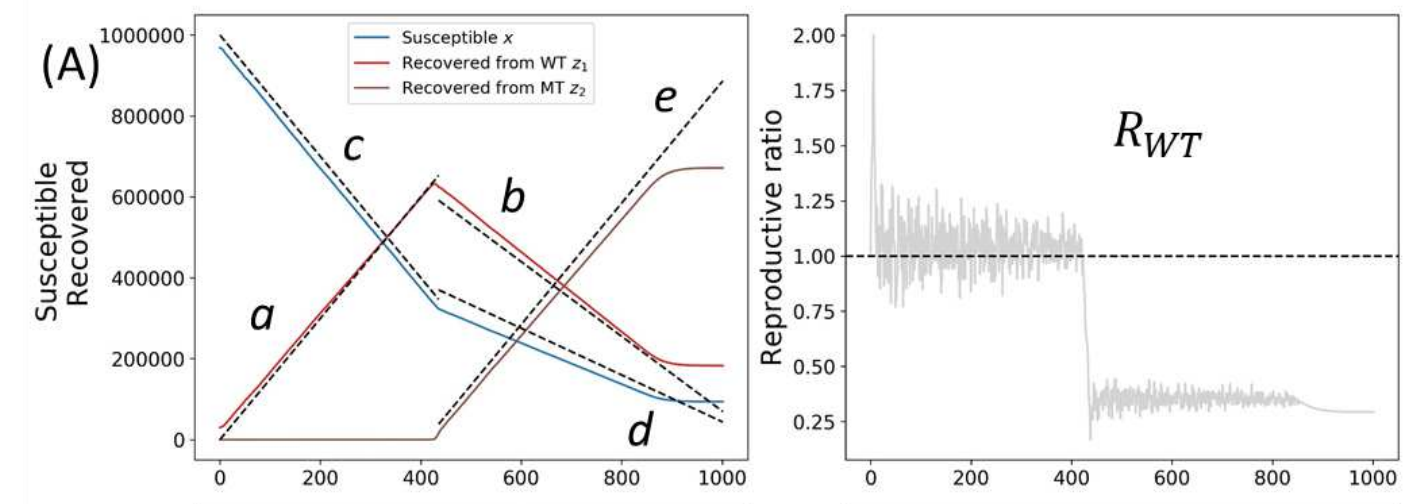

(B)
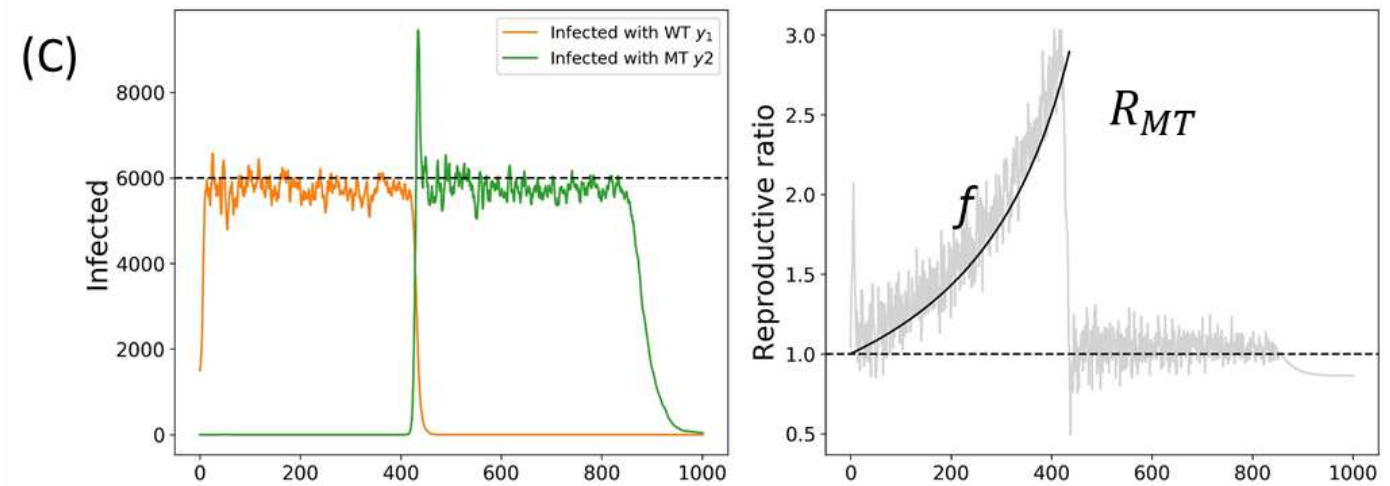

(D)
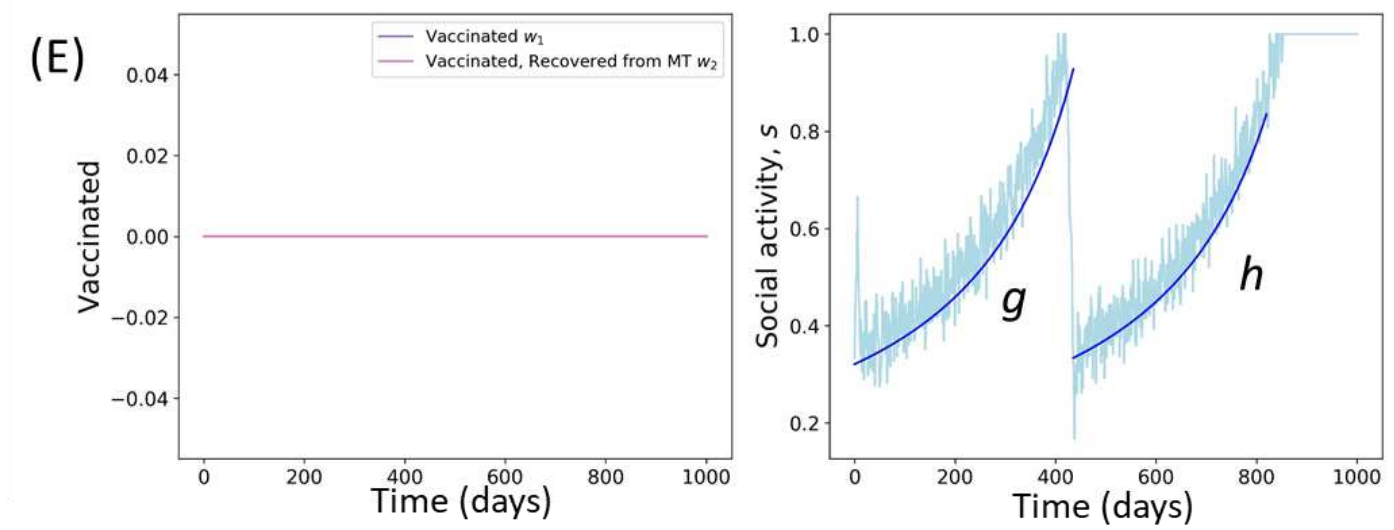

(F)

794

Figure S2: Evolution of resistance in absence of vaccination. (A) Before MT takeover, the decline in susceptible individuals $(x)$ along time can be approximated by a linear function with slope equal to $L$. Since we assume no vaccination, the number of individuals recovered from WT grows linearly with slope equal to $L$. After MT takeover, the number of individuals recovered from MT grows linearly slope equal to $L$, while the number of susceptible individuals $(x)$ and individuals recovered from WT $\left(z_{1}\right)$ declines linearly with a slope proportional to their frequencies at the moment of MT takeover. The equations of the lines (a), (b), 
801 (c), (d) and (e) are given by, with $t^{*}$ the time of takeover by the mutant strain: (a) $z_{1}(t)=z_{1}(0)+L t, t<$ $802 t^{*}(\mathrm{~b}) z_{1}(t)=\left(z_{1}\left(t^{*}\right)-z_{1}\left(t^{*}\right) /\left(z_{1}\left(t^{*}\right)+x\left(t^{*}\right)\right)\right) L\left(t^{*}-t\right), t>t^{*}$ (c) $x(t)=x(0)-L t, t<t^{*}$ (d) $x(t)=$ $803 x\left(t^{*}\right)-\left(x\left(t^{*}\right) /\left(z_{1}\left(t^{*}\right)+x\left(t^{*}\right)\right)\right) L\left(t^{*}-t\right), t>t^{*}$ (e) $z_{2}(t)=L\left(t^{*}-t\right), t>t^{*}$. (B) The reproduction 804 coefficient of the wild-type $R_{W T}$ is maintained at 1 by the dynamic lockdown. After mutant takeover, $R_{W T}$ 805 is less than 1 , since the lockdown is now adjusted to the population susceptible to the MT strain. (C) The 806 number of active cases of WT $\left(y_{1}\right)$ and after mutant takeover, $\mathrm{MT}\left(y_{1}\right)$ is constant at $L / a$ until herd immunity 807 to the MT strain is reached. (D) Before MT takeover, the reproductive rate of the MT grows as (b) $R_{M T}=$ $808 \beta_{2}\left(x(t)+z_{1}(t)\right) / a$. After takeover, $R_{M T}$ is maintained around 1. (E) In this run, there was no vaccination $809(c=0)$, hence $w_{1}=w_{2}=0$ for each time t. (F) Before MT takeover, the dynamic lockdown is adjusted to 810 the WT. As the number of individuals immune to WT grows, social activity increases. When the MT emerges, 811 lockdown measures are reinstated. Subsequently, social activity increases as the population immune to the 812 MT grows. The equations of the lines are given by (g) $s(t)=a /\left(\beta_{1} x(t)\right), t<t^{*}(\mathrm{~h}) s(t)=a /\left(\beta_{2}(x(t)+\right.$ $\left.\left.813 q z_{1}(t)\right)\right), t>t^{*}$. 


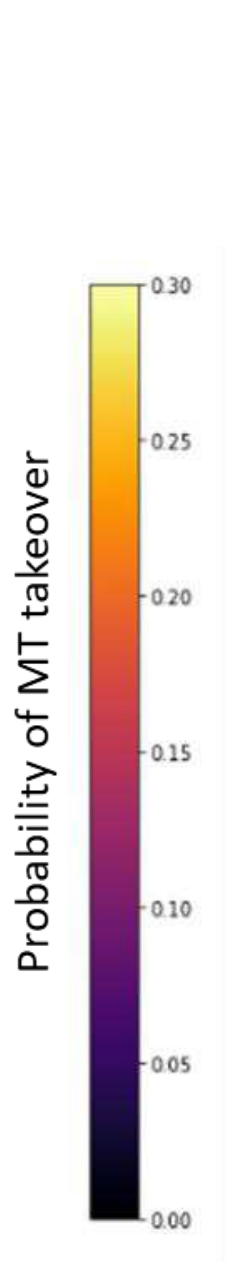

814

815

816

817

818

819

820

821 size was $N=10^{6}$.
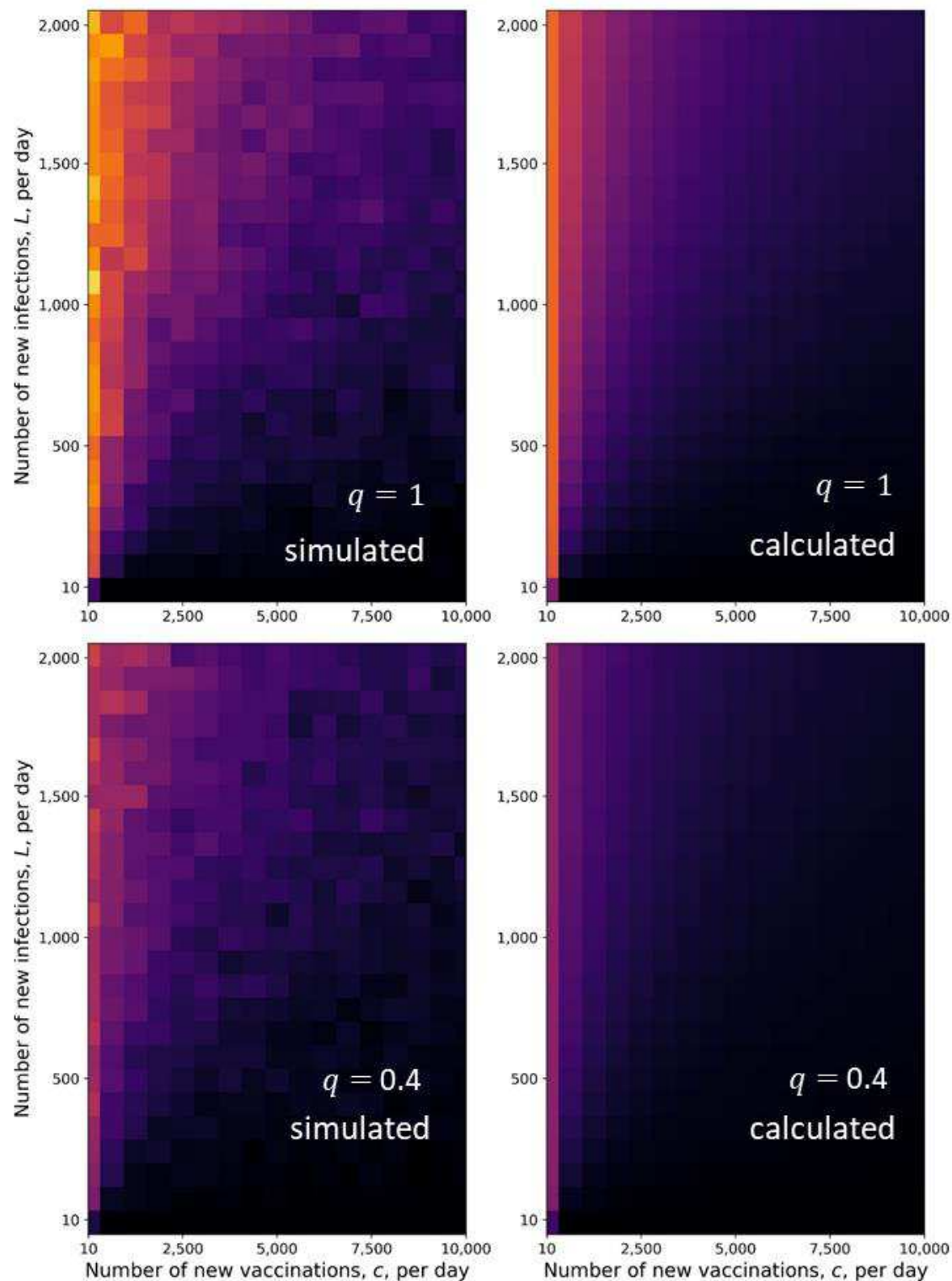

Figure S3: Analytical approximation of the simulation results. The probability of MT takeover before herd immunity is reached can be calculated according to Eq. 5. We observe a good agreement between our calculations and the results of the stochastic simulations. (A) (C) Each square of the color map is colored according to the probability of take over calculated with Eq. (5). (B) (D) Each square of the color map is colored according to the proportion of runs (out of 1000) where the MT strain took over. The population 
(A)

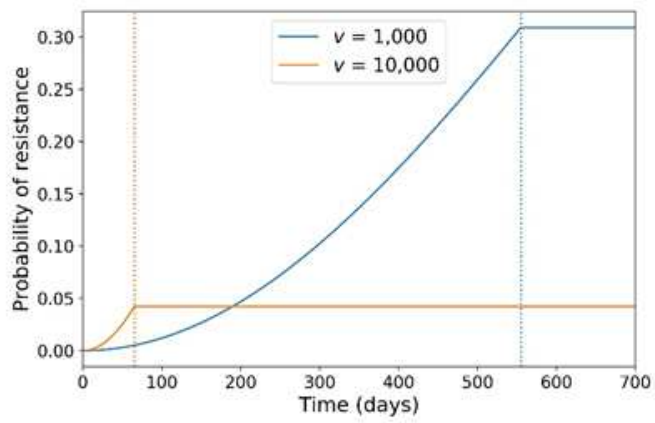

(C)

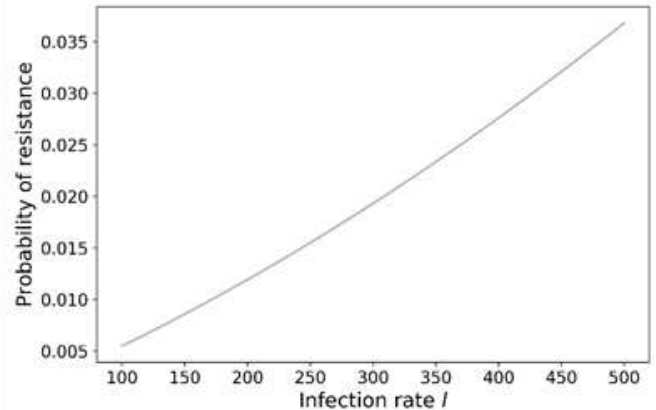

$(E)$

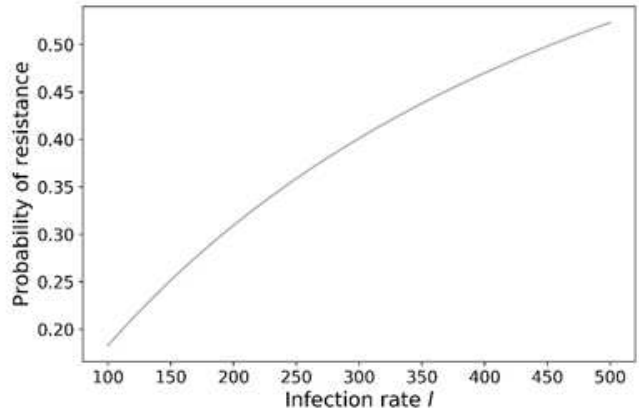

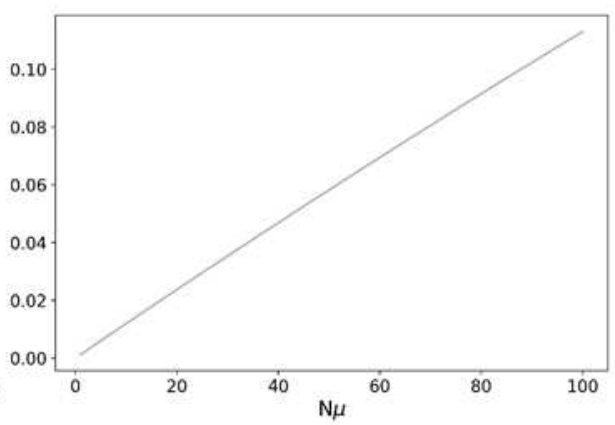

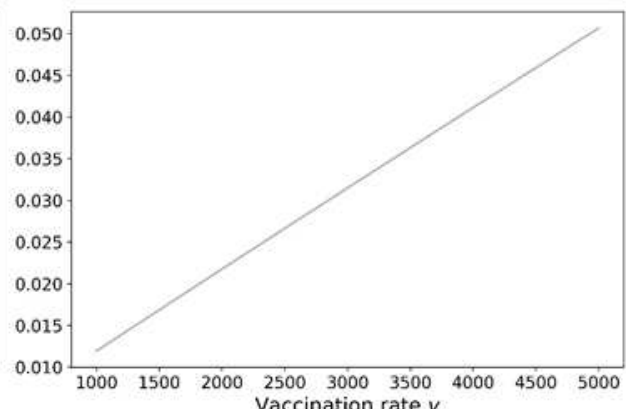

(D)

$(\mathrm{F})$

(B)

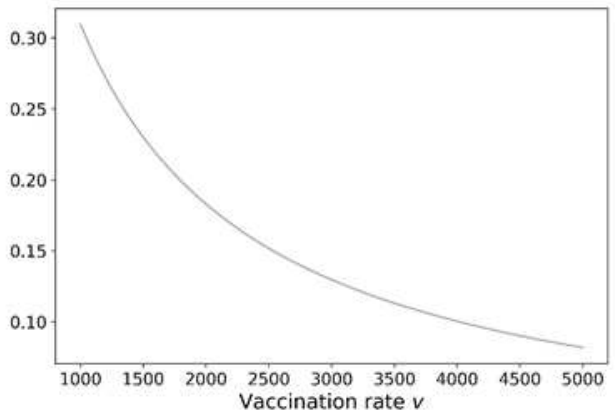

825 Figure S4: Counterintuitive effect of the vaccination rate on the probability of resistance. Increasing the

826 population size $N$, the mutation rate $\mu$ and the infection rate $l$ all increase the probability of generating a

827 mutant before herd immunity is reached (B and C). We define $v=c / N$ and $l=L / N$. Increasing the

828 vaccination rate $v$ leads to a counterintuitive effect: the probability for a fixed time increases with $v$ since

829 it leads to faster relaxation of social distancing measures, but it also leads to faster achievement of herd

830 immunity (A). Hence the probability of resistance until herd immunity decreases with $v(\mathbf{F})$. Parameter

831 values: $\mu=10^{-7}$ (A): $N=10^{8}, l=200 \cdot 10^{-6}$; (B): $l=200 \cdot 10^{-6}, v=1000 \cdot 10^{-6}$, (C)(E): $N=10^{8}, v=$ $8321000 \cdot 10^{-6} ;(\mathrm{D})(\mathrm{F}): N=10^{8}, l=200 \cdot 10^{-6}$. 